\title{
Rotation curves of high-resolution LSB and SPARC galaxies with fuzzy and multistate (ultra-light boson) scalar field dark matter
}

\author{
T. Bernal, ${ }^{1 \star} \dagger$ L. M. Fernández-Hernández, ${ }^{1} \ddagger$ T. $\operatorname{Matos}^{2} \oint \Phi$ and M. A. Rodríguez-Meza ${ }^{1} \|^{\star \star}$ \\ ${ }^{1}$ Departamento de Física, Instituto Nacional de Investigaciones Nucleares, AP 18-1027, Ciudad de México 11801, México \\ ${ }^{2}$ Departamento de Física, Centro de Investigación y de Estudios Avanzados del IPN, AP 14-740, Ciudad de México 07000, México
}

12th December 2017

\begin{abstract}
Cold dark matter (CDM) has shown to be an excellent candidate for the dark matter (DM) of the Universe at large scales, however it presents some challenges at the galactic level. The scalar field dark matter (SFDM), also called fuzzy, wave, Bose-Einstein condensate or ultra-light axion DM, is identical to CDM at cosmological scales but different at the galactic ones. SFDM forms core halos, it has a natural cut-off in its matter power spectrum and it predicts well-formed galaxies at high redshifts. In this work we reproduce the rotation curves of high-resolution low surface brightness (LSB) and SPARC galaxies with two SFDM profiles: (1) The soliton+NFW profile in the fuzzy DM (FDM) model, arising empirically from cosmological simulations of real, non-interacting scalar field (SF) at zero temperature, and (2) the multistate SFDM (mSFDM) profile, an exact solution to the Einstein-Klein-Gordon equations for a real, self-interacting SF, with finite temperature into the SF potential, introducing several quantum states as a realistic model for a SFDM halo. From the fits with the soliton+NFW profile, we obtained for the boson mass $0.212<m_{\psi} /\left(10^{-23} \mathrm{eV} / c^{2}\right)<$ 27.0 and for the core radius $0.326<r_{c} / \mathrm{kpc}<8.96$. From the combined analysis with the LSB galaxies, we obtained $m_{\psi}=0.554 \times 10^{-23} \mathrm{eV}$, a result in tension with the severe cosmological constraints. Also, we show the analytical mSFDM model fits the observations as well as or better than the empirical soliton+NFW profile, and it reproduces naturally the wiggles present in some galaxies, being a theoretically motivated framework additional or alternative to the FDM profile.
\end{abstract}

Key words: galaxies: halos, galaxies: structure, (cosmology:) dark matter

\section{INTRODUCTION}

Dark matter (DM) was postulated first in order to explain the rotation curves of disk galaxies and the observed velocity dispersions in galaxy clusters, as well as the observational mass-to-light ratios in galaxies and clusters of galaxies (Zwicky 1933, 1937; Smith 1936; Rubin et al. 1980; Rubin

* E-mail: ac13341@chapingo.mx

† Present address: Universidad Autónoma Chapingo, km. 38.5 Carretera México-Texcoco, 56230, Texcoco, Estado de México, México

$\ddagger$ E-mail: lfernandez@fis.cinvestav.mx

$\S$ E-mail: tmatos@fis.cinvestav.mx

ๆ Part of the Instituto Avanzado de Cosmología (IAC) Collaboration

|| Email: marioalberto.rodriguez@inin.gob.mx

$\star \star$ Part of the Instituto Avanzado de Cosmología (IAC) Collaboration
1983). Later, its necessity was evident to explain the gravitational lenses, the structure formation in the early Universe, the acoustic baryonic oscillations, the power spectrum of galaxies, among other astrophysical and cosmological phenomena (see e.g. Bertone et al. 2005; Bennett et al. 2013). Very recently, the space mission Planck has obtained the most precise map of the total matter-energy content of the Universe, setting the contribution of dark matter to $\sim 26 \%$, meanwhile the baryonic matter is only $\sim 5 \%$ and the cosmological constant $\Lambda$ or dark energy is $\sim 69 \%$ (Planck Collaboration et al. 2016).

The most accepted DM model is the cold dark matter $(\mathrm{CDM})$, which is very successful at reproducing the observations at cosmological scales; however, at the galactic level it faces some problems (see e.g. Weinberg et al. 2015). One of the difficulties is the so-called 'cusp/core' problem, since from CDM $N$-body simulations the DM halos are assembled with the 'cuspy' Navarro-Frenk-White density pro- 
file (Navarro et al. 1997), which is proportional to $r^{-1}$ at small radii and to $r^{-3}$ at large distances, meanwhile many observations suggest a constant central density or 'core' profile, e.g. in rotation curves of galaxies (Moore et al. 1999; de Blok et al. 2001; McGaugh et al. 2007, 2016) and dwarf spheroidal (dSph) galaxies (Klypin et al. 1999; Kroupa et al. 2010; Boylan-Kolchin et al. 2011; Walker 2013; Pawlowski et al. 2014). Another issue is the CDM prediction of too many satellite halos around big galaxies like the Milky Way (Sawala et al. 2016) which have not been observed. Aside, it fails to reproduce the phase-space distribution of satellites around the Milky Way and Andromeda galaxies (Pawlowski et al. 2012; Ibata et al. 2013, 2014) and the internal dynamics in tidal dwarf galaxies (Gentile et al. 2007; Kroupa 2012). Finally, another issue might lie in the early formation of big galaxies, since the CDM model predicts big galaxies were formed hierarchically from halos less massive than $10^{12} M_{\odot}$, as is the typical case, but there are some recent observations of massive galaxies at very high redshifts $z(5 \leqslant z<6)$ (Caputi et al. 2015).

Such problems at the galactic scales might be solved by introducing the baryonic physics to the simulations, which can be relevant at the centers of the galaxies and galaxy clusters through the inclusion, for example, of star formation, supernova explosions, stellar winds, active galactic nuclei, dynamical friction, etc. (see e.g. El-Zant et al. 2001, 2004; Ma \& Boylan-Kolchin 2004; Nipoti et al. 2004; Romano-Díaz et al. 2008; Del Popolo 2009; Governato et al. 2010; Del Popolo 2012a,b; Teyssier et al. 2013; Pontzen \& Governato 2012; Madau et al. 2014; Di Cintio et al. 2014; Pontzen \& Governato 2014; Nipoti \& Binney 2015; Del Popolo \& Le Delliou 2017). From a phenomenological point of view, empirical CDM density profiles have been proposed in order to explain the observations, e.g. the Burkert profile (Burkert 1996; Salucci \& Burkert 2000) and the generalized NFW profile (Zhao 1996). Aside, there are some alternatives to CDM seeking to solve the discrepancies at galactic scales without appealing to baryonic processes, for instance, warm dark matter (Zavala et al. 2009; Navarro et al. 2010; Lovell et al. 2012), self-interacting dark matter (Spergel \& Steinhardt 2000; Yoshida et al. 2000; Davé et al. 2001; Elbert et al. 2015; Robles et al. 2017) and scalar field dark matter (SFDM) (see Matos \& Guzman 2000; Magaña et al. 2012; Suárez et al. 2014; Matos \& Robles 2016; Marsh 2016; Hui et al. 2017, and references therein).

We are interested in the SFDM model, studied within some special cases and named differently depending on the authors. The motivation is the natural solution emerging from this model to the CDM problems mentioned before. The SFDM model assumes DM is a spin-0 scalar field (SF) $\psi$, with a typical ultra-light mass $m_{\psi} \sim 10^{-23}-10^{-21} \mathrm{eV} / c^{2}$, which might include self-interactions. The first time when this idea was mentioned was in Baldeschi et al. (1983); since then the idea was rediscovered several times using different names (see e.g. Membrado et al. 1989; Press et al. 1990; Sin 1994; Ji \& Sin 1994; Lee \& Koh 1996; Matos \& Guzman 2000; Sahni \& Wang 2000; Peebles 2000; Goodman 2000; Matos \& Ureña-López 2000, 2001; Hu et al. 2000; Wetterich 2001; Arbey et al. 2001; Boehmer \& Harko 2007; Matos et al. 2009; Woo \& Chiueh 2009; Lundgren et al. 2010; Bray 2010; Marsh \& Ferreira 2010; Robles \& Matos 2013b; Schive et al. 014a) and more recently in Hui et al. (2017). However, the first systematic study of this idea started in 1998 by Guzman et al. (1999); Matos \& Guzman (2000), showing that the observed rotation curves of disk galaxies can be reproduced by the SFDM model, and the cosmology was studied for the first time in Matos et al. (2000); Matos \& UreñaLópez (2000). Other systematic studies of the SFDM model were performed by Arbey et al. (2001); Arbey et al. (2002) and more recently in Marsh \& Ferreira (2010); Schive et al. (014a); Marsh (2016).

In Matos et al. (2000); Matos \& Ureña-López (2000), using the amount of satellite galaxies observed in the vicinity of the Milky Way, it was found that the mass of the SF should be $m_{\psi} \sim 10^{-22} \mathrm{eV} / c^{2}$ (see also Hernández-Almada et al. 2011; Rodríguez-Meza et al. 2012, for the estimation of the SF mass from a Jeans' stability analysis, $m_{\psi}$ $\left.\sim 10^{-22} \mathrm{eV} / c^{2}\right)$. With this ultra-light mass, Alcubierre et al. (2002) found, through numerical simulations, that the gravitational collapse of a SF configuration forms stable objects with mass $M$ of the order of a galaxy halo, $M \sim 10^{12} M_{\odot}$. Also, with the characteristic ultra-light SF mass, the bosons condense very early in the Universe at critical condensation temperatures of $\mathrm{TeV}$ (Matos \& Ureña-López 2001), making up Bose-Einstein condensates (BEC) interpreted as the DM halos. One important property of the SFDM is that, at cosmological scales, it behaves as dust and reproduces the same observations as well as CDM: the cosmic microwave background $(\mathrm{CMB})$ and the mass power spectrum at large scales (Rodríguez-Montoya et al. 2010; Hlozek et al. 2015; Schive et al. 2016). Recently, Schive et al. (014a,b) have run high-resolution cosmological simulations of SFDM and reproduced the same results.

In a series of papers (Matos \& Ureña-López 2007; Bernal et al. 2008; Robles \& Matos 2012) it was shown that the SFDM forms core halos (see also Harko 2011). Further features of the SFDM have been analyzed, for example, the gravitational lensing (Núñez et al. 2010; Robles \& Matos 2013a), the $\psi^{4}$-SF potential (e.g. Matos \& Suarez 2011; Matos et al. 2016), multistate SF solutions, i.e. bosons in excited states outside the ground energy level (see e.g. Bernal et al. 2010; Robles \& Matos 2013b; MartinezMedina et al. 2015a; Bernal et al. 2017). Numerical simulations of galaxy formation were performed (Martinez-Medina \& Matos 2014; Martinez-Medina et al. 2015b), where the characteristic spiral arms and bars of a disk galaxy were easily generated. It was also shown that satellite galaxies are stable around SFDM halos (Robles et al. 2015). The hydrodynamical version of the SF equations of motion were first shown in Hernández-Almada et al. (2011); RodríguezMeza et al. (2012), where it was demostrated that vorticity is present in this 'fluid'. Also a study of high-resolution rotation curves of spiral galaxies in the BEC-DM and flatoscillaton models has been done (Fernandez-Hernandez et al. 2017). And there are some works investigating the possibility that galactic ultra-light boson halos may host and even collapse into the supermassive black-holes observed in many galaxies (Chavanis 2016; Avilez et al. 2017).

As mentioned before, the SFDM model has many variants, depending on the specific characteristics studied by different authors: the SF might be real or complex and possess a self-interaction, it might take into account also the temperature of the SF, by introducing a suitable SF potential. The SFDM model has been named as fuzzy DM (FDM) 
(Hu et al. 2000), wave DM (WDM or $\psi$ DM) (Bray 2012; Schive et al. 014a), ultra-light axion (ULA) DM (Hlozek et al. 2015), Bose-Einstein condensate (BEC) DM (Boehmer \& Harko 2007; Fernandez-Hernandez et al. 2017), multistate SFDM (mSFDM) (Robles \& Matos 2013b), etc.

In the BEC-DM model (Boehmer \& Harko 2007) it is assumed a dominant self-interaction between the particles (Thomas-Fermi limit); in this approximation all the bosons lie in the ground state at zero temperature; the mean boson mass from the analytic density profile is $m_{\psi} \sim 10^{-6} \mathrm{eV} / c^{2}$. This model has been widely studied, showing up sharp discrepancies in dwarf galaxies (Diez-Tejedor et al. 2014), disk galaxies (Boehmer \& Harko 2007; Robles \& Matos 2012; Fernandez-Hernandez et al. 2017) and galaxy clusters (Bernal et al. 2017), ruling out this approximation as a realistic SFDM model.

The first model studied in the present work is the fuzzy, wave or ultra-light axion DM, which share the same characteristics: a quadratic non-thermal, non-interacting potential with ultra-light masses, $m_{\psi} \sim 10^{-23}-10^{-21} \mathrm{eV} / c^{2}$. From the cosmological simulation by Schive et al. (014a,b), an empirical density profile was obtained for this model, composed by a coupling of the asymptotic NFW decline with an inner soliton-like profile from the SFDM ('soliton+NFW' profile) (Schive et al. 014a; Marsh \& Pop 2015).

Secondly, we investigate the multistate SFDM model (Robles \& Matos 2013b), that considers the SF is thermal at the very early Universe, interacting with the radiation and the rest of matter. By introducing a self-interacting, temperature-corrected SF potential, the authors obtained an exact analytic solution for the SF density, including the ground and excited quantum states. This model has been proved successful in fitting the observations of dwarf spheroidal galaxies (Martinez-Medina et al. 2015a), the rotation curves of disk galaxies (Robles \& Matos 2013b), the strong gravitational lensing (Robles \& Matos 2013a) and the dynamical masses from X-ray observations of clusters of galaxies (Bernal et al. 2017). Observe that both, the soliton+NFW and the mSFDM profiles are approaches of the same model for galactic applications, the difference lies in that the first one comes from numerical simulations, and the second one comes from approximations of the field equations but with finte temperature in the SF potential.

In this work, we fit the observed rotation curves of 18 high-resolution low surface brightness (LSB) galaxies, where only the DM is taken into account, 4 representative SPARC (Spitzer Photometry \& Accurate Rotation Curves) galaxies and 2 other NGC galaxies used in Robles \& Matos (2013b) (the last 6 galaxies with high-resolution photometric information). For the galaxies with baryonic data, McGaugh et al. (2016); Lelli et al. (2017) analyzed 153 galaxies with the gas and stars information from the SPARC database (Lelli et al. 2016), and found an empirical radial acceleration relation between the observed acceleration from the rotation curves of the galaxies and the acceleration from the baryonic component, showing a deviation at the value $g^{\dagger}=1.2 \times 10^{-10} \mathrm{~m} / \mathrm{s}^{2}$. This result could suggest that baryons are the source of the gravitational potential, at least at small radii. As McGaugh et al. (2016) pointed out, such relation can be explained as the end product of galaxy formation processes (including the baryonic matter), new DM physics or the result of a modified gravity law. In the CDM paradigm,
Ludlow et al. (2017) introduced different stellar and AGN feedback processes to explain the observed acceleration relation in well-resolved galaxies from the EAGLE simulation, showing that the empirical relation can be 'accommodated' within the model. However, instead to look for complicated and diverse baryonic processes, in this work we test the two SFDM models with the observed rotation curves of some representative SPARC galaxies, showing that the model is consistent with the data. The results given here will give us a clue for the next generation of numerical simulations. The soliton+NFW profile does not contain finite temperature while the mSFDM profile is not a numerical result. The best SFDM profile will be the one that takes these two ingredients into account.

The idea is to analyze statistically both approximations: the study can give us a clue for whether the scalar field is thermal or not, or if the self-interaction between SF particles can be important, assuming the scalar field is the dark matter of the galaxies. In order to do so, this work is organized as follows: In Section 2 we briefly explain the SFDM model and the two different approaches to be compared; in Section 3 we describe the galaxies' samples used to fit the SFDM models; in Section 4 we present the results, and in Section 5 we discuss the results and present our conclusions.

\section{SCALAR FIELD DARK MATTER}

From particle physics motivation, spin-0 scalar fields are the simplest bosonic particles, described by the Klein-Gordon (KG) equation:

$\square \psi-\frac{\mathrm{d} V(\psi)}{\mathrm{d} \psi}=0$,

for the real or complex scalar field $\psi$ and the SF potential $V(\psi)$; from hereafter we use the notation $\hbar=1=c$, for $c$ the speed of light and $\hbar$ the reduced Planck's constant. The scalar fields arise in cosmology to explain the inflationary epoch at the very early Universe and as alternatives to explain the accelerated expansion. They can describe also compact objects, like boson stars and supermassive black holes. As alternative to the CDM paradigm, it is proposed that DM halos are constituted by ultra-light scalar fields, for which the complete Einstein-Klein-Gordon equations reduce to the Schrödinger-Poisson system in the Newtonian limit.

Until now, there is not an agreement on the correct form of the potential $V(\psi)$ for DM applications and some of them have been proposed in order to apply the SFDM theory to different astronomical and cosmological situations (see e.g. Suárez et al. 2014). A general potential is given by

$V(\psi)=\frac{1}{2} m_{\psi}^{2} \psi^{2}+\frac{1}{4} \lambda \psi^{4}$,

the so-called 'double-well' potential, which includes the mass $m_{\psi}$ of the SF and a self-interaction $\lambda$. For positive $m_{\psi}^{2}$ the potential has a single minimum at $\psi=0$ and the potential is $Z_{2}$ invariant; for $-m_{\psi}^{2}$ the potential has two minima and the $Z_{2}$ symmetry appears spontaneously broken, being of great interest for physical situations (see Subsection 2.2). A recent study of large scale structure formation with the last potential has been developed in Suárez \& Chavanis (2017). 
For this potential, in the hydrodynamic approach, it is possible to write an equation of state of the SF, resulting in a polytrope of index $n=1$.

In the case $\lambda=0$, the potential (2) reduces to

$V(\psi)=\frac{1}{2} m_{\psi}^{2} \psi^{2}$,

which has been widely used to model the SFDM in the Universe, as discussed in the next Subsection.

\subsection{Fuzzy (wave) dark matter}

The fuzzy DM (FDM) (Hu et al. 2000), wave DM (WDM or $\psi \mathrm{DM}$ ) (Bray 2012; Schive et al. 014a) or ultra-light axion (ULA) DM (Hlozek et al. 2015) (here we name it as Hu et al. (2000), FDM), considers the SFDM is described by the potential (3), for ultra-light masses $m_{\psi} \sim 10^{-23}-10^{-21} \mathrm{eV}$ and null self-interaction $\lambda=0$. As showed in Matos \& Ureña-López (2001) (see also Schive et al. 014a; Ureña-López \& González-Morales 2016), this approximation presents a cut-off in the power spectrum which suppresses the small scale structure formation below the de Broglie wavelength $\lambda_{\mathrm{deB}}$ (corresponding to halo masses $M<10^{8} M_{\odot}$ for $m_{\psi} \sim$ $\left.10^{-22} \mathrm{eV}\right)$, as a result of the quantum properties of the model (Heisenberg uncertainty principle), solving in this way the small scale structure overproduction in CDM. This approach assumes the SFDM is at zero temperature, implying all the bosons are in the ground state, i.e. the lowest energy level with no nodes. Also, Suarez \& Matos (2011) showed that, within this approach, the evolution of perturbations of the SFDM model is identical to $\Lambda$ CDM and Rodríguez-Montoya et al. (2010) showed the SFDM model is consistent with the acoustic peaks of the CMB for a boson mass $m_{\psi} \sim 10^{-22} \mathrm{eV}$.

Nevertheless, Li et al. (2014) found that this model is not consistent with the big bang nucleosynthesis (BBN) constraints for the boson mass $m_{\psi}$ for any model with $\lambda=0$, at $1 \sigma$-confidence level (CL), making necessary to introduce a self-interaction into the SF potential. Although their results allow to alleviate such restrictions at $2 \sigma$-CL, their work is a motivation to study a SF potential including a non-null self-interaction (see Subsection 2.2).

Schive et al. (014a,b) run a high-resolution cosmological simulation based on the dynamics of FDM in the Newtonian limit, governed by the Schrödinger-Poisson system (see also Schwabe et al. 2016; Mocz et al. 2017). From the simulation, Schive et al. (014a) derived an empirical density profile for the DM halos, consisting of a soliton-like core in every system, prominent before a transition radius, embedded in a NFW density halo dominant at large radii. The motivation of this proposal is the expected loss of phase coherence of the FDM waves at large distances from the center of distribution, expecting a transition from the soliton to the NFW profile (Marsh \& Pop 2015). Such profile can be approximated by

$\rho_{\mathrm{FDM}}(r)=\Theta\left(r_{\epsilon}-r\right) \rho_{\mathrm{sol}}(r)+\Theta\left(r-r_{\epsilon}\right) \rho_{\mathrm{NFW}}(r)$,

where $\Theta$ is a step function, $r_{\epsilon}$ is the transition radius where the density changes from the soliton profile (Schive et al. 014a)

$\rho_{\mathrm{sol}}(r)=\frac{\rho_{c}}{\left[1+0.091\left(r / r_{c}\right)^{2}\right]^{8}}$, to the NFW profile (Navarro et al. 1997)

$\rho_{\mathrm{NFW}}(r)=\frac{\rho_{s}}{\left(r / r_{s}\right)\left(1+r / r_{s}\right)^{2}}$.

In equation (5), $\rho_{c}:=1.9\left(m_{\psi} / 10^{-23} \mathrm{eV}\right)^{-2}\left(r_{c} / \mathrm{kpc}\right)^{-4} M_{\odot} \mathrm{pc}^{-3}$ is the central soliton density, $m_{\psi}$ the boson mass and $r_{c}$ the half-light radius of the soliton-like region. In equation (6), $\rho_{s}$ is related to the density of the Universe at the moment the halo collapsed and $r_{s}$ is a scale radius. The total density profile (4) has five free parameters $\left(m_{\psi}, r_{c}, r_{\epsilon}, \rho_{s}, r_{s}\right)$, which are reduced to four asking for continuity of the function at the transition radius $r_{\epsilon}$ (see Appendix A). From the cosmological simulations, Schive et al. (014a) found that the transition radius $r_{\epsilon}$ is, in general, $r_{\epsilon}>3 r_{c}$.

There are several constraints to the boson mass $m_{\psi}$ in the FDM model: In the cosmological context, from CMB and galaxy clustering data,Hlozek et al. (2015) constrained the mass $m_{\psi}>10^{-24} \mathrm{eV}$. From the galaxy UV-luminosity function and reionization constraints, Bozek et al. (2015) obtained $m_{\psi}>10^{-23} \mathrm{eV}$, and from the high-redshift luminosity function of galaxies, Schive et al. (2016) get $m_{\psi}>1.2 \times$ $10^{-22}$ eV. From Lyman- $\alpha$ observations, Sarkar et al. (2016) obtained $m_{\psi}>10^{-23} \mathrm{eV}$, and very recently, Iršič et al. (2017); Armengaud et al. (2017) derived $m_{\psi} \gtrsim 2 \times 10^{-21} \mathrm{eV}$, providing a severe constraint to smaller masses.

From astrophysical observations, the study of the effect of tidal forces on the cold clumps in Ursa Minor, Lora et al. (2012) found $m_{\psi} \sim(0.3-1) \times 10^{-22} \mathrm{eV}$, and in Sextants, Lora \& Magaña (2014) found $m_{\psi} \sim(0.12-8) \times 10^{-22} \mathrm{eV}$. From ultra-faint dwarf spheroidal (dSph) galaxies, Calabrese \& Spergel (2016) obtained $m_{\psi} \sim(3.7-5.6) \times 10^{-22} \mathrm{eV}$. For the classical dSph galaxies in the Milky Way, Marsh \& Pop (2015) constrained $m_{\psi}$ using the soliton+NFW density profile (4), through stellar populations in Fornax and Sculptor and found $m_{\psi}<1.1 \times 10^{-22} \mathrm{eV}$. Chen et al. (2017) applied the Jeans analysis to the kinematic data of the eight dSphs to constrain $m_{\psi}$, where, for all the dSphs except for Fornax, as the transition radii are outer the half-light radii $r_{c}$, they took into account the soliton core profile (5) only, deriving a mass $m_{\psi}=1.18 \times 10^{-22} \mathrm{eV}$; for Fornax, they applied the complete density profile (4) and found a larger $m_{\psi}=1.79 \times 10^{-22} \mathrm{eV}$ and smaller $r_{c}$, with respect to the soliton-only model results. González-Morales et al. (2016) constrained $m_{\psi}$ with the complete soliton+NFW profile (4), using kinematic mock data of Fornax and Sculptor, that include the stellar components of the galaxies, finding core radii $r_{c}>1.5 \mathrm{kpc}$ and $r_{c}>1.2 \mathrm{kpc}$, respectively, and $m_{\psi}<4 \times 10^{-23} \mathrm{eV}$; the authors propose that such small boson mass might be the result of baryonic feedback processes present in the dSph galaxies. Studying a set of LSB rotation curves, Garcia-Aspeitia et al. (2017) showed that FDM gives values of $M \sim 10^{7} M_{\odot}$ within $300 \mathrm{pc}$, which is consistent with results found in Strigari et al. (2008) for satellite galaxies in the Milky Way.

And very recently, Ureña-López et al. (2017) proposed a way to determine the boson mass using the results from the mass discrepancy-acceleration relation presented in McGaugh et al. (2016); Lelli et al. (2016) from the observations in the SPARC catalog, that implies a universal maximum acceleration for all the galaxy halos, and found a relation between the soliton core radius $r_{c}$, the boson mass $m_{\psi}$ and the central surface density of the galaxies. With their analysis with the soliton profile, they found that the 
dwarf galaxies in the Milky-Way and Andromeda are consistent with a boson mass $m_{\psi} \approx 1.2 \times 10^{-21} \mathrm{eV}$, in agreement with the cosmological constraint $m_{\psi}>10^{-23} \mathrm{eV}$.

Therefore, we have the lower limit $m_{\psi}=10^{-23} \mathrm{eV}$ (Bozek et al. 2015; Sarkar et al. 2016), and from the latest works with Lyman- $\alpha$ data the limit $m_{\psi} \approx 2 \times 10^{-21} \mathrm{eV}$ (Iršič et al. 2017; Armengaud et al. 2017). Taking into account the last result, there would be a tension with many of the constraints mentioned before, coming from diverse observations. At this point, there is not an agreement on the correct mass of the ultra-light boson, and in this article, we explore the viability of the FDM model in galaxies of many sizes to report the boson mass needed to reproduce the observed rotation curves.

For the LSB and SPARC galaxies analyzed in the present work, we assume the soliton+NFW profile (4) and expect the transition radius $r_{\epsilon}$ is well inside the radius of the last observed point (spanning a range $R_{\max } \sim 1-30 \mathrm{kpc}$, for the maximum radius of luminous matter), thus we apply the complete density profile. A continuity condition between the soliton and NFW profiles at the transition radius $r_{\epsilon}$ is imposed, assuming an abrupt transition between the soliton and the external NFW halo, as found in the cosmological simulations (Schive et al. 014a; Schwabe et al. 2016; Mocz et al. 2017). With this condition, it is possible to write the NFW parameter $\rho_{s}$ in function of the other four free parameters, thus we fit only $m_{\psi}, r_{c}, r_{\epsilon}$ and $r_{s}$. The complete expressions are written in Appendix A.

\subsection{Multistate scalar field dark matter}

As a step forward on the study of the SFDM model, Robles \& Matos (2013b) considered the general double-well potential (2), with a non-null self-interaction term, plus one-loop finite-temperature $T$ corrections (Kolb \& Turner 1994):

$V(\psi)=-\frac{1}{2} m_{\psi}^{2} \psi^{2}+\frac{1}{4} \lambda \psi^{4}+\frac{1}{8} \lambda \psi^{2} T^{2}-\frac{\pi^{2}}{90} T^{4}$,

in units $k_{\mathrm{B}}=1$ for the Boltzmann constant and where the $Z_{2}$ symmetry appears spontaneously broken (with the term $\left.-m_{\psi}^{2}\right)$. Such self-interacting, finite-temperature corrected potential is motivated by the theoretical expectation of modern particle physics that at high temperatures symmetries spontaneously broken today were restored. Symmetry breaking studies are of great interest for diverse physical situations, assuming the Universe has underwent phase transitions during its evolution (see e.g. Kolb \& Turner 1994, and references therein), as for example, the inflationary era. With this motivation in mind, Robles \& Matos (2013b) considered that, at the very early Universe, the initial SF fluctuations from inflation interact with the rest of matter and radiation at very high temperatures $T \gg T_{c}$, for $T_{c}$ the critical temperature where $\psi=0$ is a minimum of the potential and the symmetry is restored. In this case, the SF is embedded in a thermal bath at finite temperature $T$ at very early times ${ }^{1}$. As the Universe expands, the temperature decreases and eventually the SF decouples from the rest of matter,

${ }^{1}$ In equation (7), the term $\propto \psi^{2} T^{2}$ appears due to the interaction of the SF with the thermal bath and the term $\propto T^{4}$ is due to the thermal bath only. evolving independently. As the potential (7) depends on the 4 th-power with respect to $T$, as the temperature continues decreasing, the $\mathrm{SF}$ goes through a $Z_{2}$ spontaneous symmetry breaking (SSB), which turns a minimum of the potential to a maximum, that increases the amplitude of the initial SF perturbations forming the initial galaxy halos (see also Matos \& Rodríguez-Meza 2014, for the study of the SSB of a charged complex SF).

For cosmological applications, Robles \& Matos (2013b) assumed the SSB at $T=T_{c}$ takes place in the radiation dominated era. They found that at that moment, the SF fluctuations can start growing in the linear regime for $T<T_{c}$, until they reach a new stable minimum. Then obtained the perturbed Einstein-Klein-Gordon evolution equations for the SF perturbation, $\delta \psi$, in a Friedmann-Lemaître-RobertsonWalker background space-time. Under these assumptions, the galactic halos could have been formed almost at the same time of the SSB and with similar masses, $M \sim 10^{12} M_{\odot}$ (for $m_{\psi} \sim 10^{-22} \mathrm{eV}$ ), which is the typical mass of a galaxy like ours. Later on, such halos can enter in the non-linear regime, merging and constituting larger structures, hierarchically, just like in the CDM model.

Under the linear approximation to describe the evolution of a galaxy halo, in the Newtonian regime, the exact solution to the perturbation equations for the temperaturecorrected potential (7) is found as (Robles \& Matos 2013b)

$\rho_{\mathrm{mSFDM}}^{j}(r)=\rho_{0}^{j}\left[\frac{\sin \left(k_{j} r\right)}{\left(k_{j} r\right)}\right]^{2}$,

whose mass distribution is given by

$M_{\mathrm{mSFDM}}^{j}(r)=\frac{4 \pi \rho_{0}}{k_{j}^{2}} \frac{r}{2}\left[1-\frac{\sin \left(2 k_{j} r\right)}{\left(2 k_{j} r\right)}\right]$.

This solution is naturally core. Thus, the finite-temperature corrected potential (7) implies the existence of different excitation states $j$ as solution to the SF perturbation equation, i.e. the bosons are thermally distributed in the ground and excited states at higher energy levels, and the general solution allows a configuration out of the fully condensate system. In the last equation, $j=1,2,3, \ldots$ is the number of the excited state required to fit the mass distribution, $\rho_{0}^{j}=\rho_{\mathrm{mSFDM}}^{j}(0)$ is the central density and the radius $R$ of the SF configuration is fixed through the condition $\rho_{\mathrm{mSFDM}}^{j}(R)=0$, i.e. $k_{j} R=j \pi$.

Notice that when using the analytic profile (8) there is a degeneracy in the function, since if we keep the ratio $j / R$ constant, the form of the density profile does not change, only the state $j$ and radius $R$ become bigger. The radius $R$ is chosen as the minimum radius required to fit the last observational point and it is defined as the radius of the halo, even though the realistic SFDM halo goes to infinity. In this approximation, to fit diverse observations, the oscillations for $r>R$ are not taken into account given that they decay very quickly with $r$.

Now, as the solution to the SF perturbation equation is linear, it is possible to have combinations of excited states; this means that the total density $\rho_{\text {mSFDM }}$ can be written as the sum of the densities in the different excited states (Robles \& Matos 2013b):

$\rho_{\mathrm{mSFDM}}(r)=\sum_{j} \rho_{0}^{j}\left[\frac{\sin (j \pi r / R)}{(j \pi r / R)}\right]^{2}$. 
The last equation corresponds to the multistate SFDM (mSFDM) model. It is worth noting that the radius $R$ in the last equation is the same for all the excited states present in the configuration and, as explained before, it is defined as the radius of the mSFDM halo. Such profile has at least three free parameters for one state and every additional excited state adds two parameters more, $j$ and $\rho_{0}^{j}$.

This theoretically motivated SFDM profile has a big disadvantage: the physical parameters of the SF, like the boson mass $m_{\psi}$ and self-interaction parameter $\lambda$, are degenerated with other quantities, like the critical temperature $T_{c}$ at the $\mathrm{SSB}$, the temperature of the halo at the formation time, etc., thus it is not possible to constrain the properties of the boson particle with only one independent observation. There are some works trying to constrain the self-interaction for a $\psi^{4}$-potential (see e.g. Li et al. 2014, 2016; Suárez \& Chavanis 2017 ). However, the mSFDM model has been proved successful in fitting the observations of dwarf spheroidal galaxies (Martinez-Medina et al. 2015a), the rotation curves of galaxies (Robles \& Matos 2013b), the dynamical masses from Xray observations of clusters of galaxies (Bernal et al. 2017), in predicting the size of the Einstein radius of lensed galaxies by strong gravitational lensing (Robles \& Matos 2013a) and the survivability of satellite SFDM halos orbiting around a Milky Way-like galaxy (Robles et al. 2015). At this point, the mSFDM profile can be used as a useful fitting function and along with or as an analytic alternative to the empirical soliton+NFW density profile, as disscused below.

\section{GALAXIES' SAMPLES}

\subsection{High-resolution LSB galaxies}

We are using two sets of galaxy rotation curves according to the availability of photometric data. For the low surface brightness (LSB) galaxies, in which the dark matter is the dominant component, we are using the observed highresolution rotation curves of $18 \mathrm{LSB}$ galaxies reported in de Blok et al. (2001). In that work, the authors considered the visible data contribution, classifying the galaxies according with the availability of photometric data and using three models: the minimum disk model, where the dark matter is the principal component in the halos making zero all the visible components for the galaxies with photometry; a constant $M / L_{*}$ model, and the maximum disk model, that considers the galaxies with photometric data only.

For the first part of the work, we used the set of the LSB galaxies without photometric data, where the DM is the dominant component. This set is described in Table 1, where we present some important characteristics of each galaxy.

\subsection{NGC galaxies with photometric data}

In the second place we selected, as representative systems of the set of galaxies with photometric data, the three sample galaxies reported in McGaugh et al. (2016) (NGC 7814, 6503, 3741) from the SPARC (Spitzer Photometry \& Accurate Rotation Curves) database (Lelli et al. 2016), and the three sample galaxies used in Robles \& Matos (2013b) (NGC 1003, data from SPARC; NGC 1560, data from de
Blok et al. (2001); NGC 6946, data from McGaugh (2005)). The characteristics of these galaxies are shown in Table 2.

The SPARC database contains the near-infrared photometry (tracing the stellar mass distribution) and highresolution $\mathrm{HI} / \mathrm{H} \alpha$ rotation curves (tracing the gravitational potential out to large radii) of 175 disk galaxies, deriving with high resolution the stellar and gas components of the galaxies (Lelli et al. 2016). The sample represents a wide range in morphological types, stellar masses, surface brightnesses and gas fractions, and the mass models are reconstructed from the observed distributions of stars and gas for different characteristic radii and values of the stellar massto-light ratio. The database includes low surface brightness (LSB) galaxies, in major part gas-dominated dwarf galaxies; high surface brightness (HSB) galaxies, in major part bulgedominated spiral galaxies; and intermediate surface brightness (ISB) galaxies, which are disk-dominated systems.

Usually, in LSB galaxies the DM component is dominant even at small radii, whilst in HSB galaxies the contribution of the stellar and gas components to the total mass is important at the central regions. However, McGaugh et al. (2016) found that, even when the dark matter is dominant at the inner regions for many galaxies, the observed acceleration in 153 galaxies of all types (LSB, ISB and HSB galaxies) from the SPARC database, strongly correlates with the acceleration from the baryonic matter, showing a mass discrepancy at the value $g^{\dagger}=1.2 \times 10^{-10} \mathrm{~m} / \mathrm{s}^{2}$. In this work, we investigate the consistency of the two SFDM models with the observational rotation curves of four representative SPARC galaxies, including the baryonic information.

\subsection{Statistical calibration method}

The galaxy rotation curve is given by

$V(r)=\sqrt{\frac{G M_{T}(r)}{r}}$,

where $M_{T}=M_{\text {bulge }}+M_{\text {disk }}+M_{\text {gas }}+M_{\mathrm{DM}}$, for $M_{\mathrm{DM}}$ the dark matter contribution depending on the density profile used (soliton+NFW or mSFDM). In the case of the LSB galaxies (Subsection 3.1), $M_{T}=M_{\mathrm{DM}}$, and for the galaxies with photometric information (Subsection 3.2) we considered the different baryonic components (bulge, disk-stars and gas), if given.

In order to constrain the free parameters of every SFDM model, we used the Markov Chain Monte Carlo (MCMC) method (Gamerman 1997), through a maximization of the likelihood function $\mathcal{L}(\mathbf{p})$ given by

$\mathcal{L}(\mathbf{p})=\frac{1}{(2 \pi)^{N / 2}|\mathbf{C}|^{1 / 2}} \exp \left(-\frac{\boldsymbol{\Delta}^{T} \mathbf{C}^{-1} \boldsymbol{\Delta}}{2}\right)$,

where $\mathbf{p}$ is the vector of parameters, $N$ the number of observational points for each galaxy, $\boldsymbol{\Delta}=V_{\mathrm{obs}}\left(r_{i}\right)-V_{\text {model }}\left(r_{i}, \mathbf{p}\right)$, for $V_{\text {obs }}$ the observational circular velocity at the radius $r_{i}$ and $V_{\text {model }}$ the derived total velocity for a given SFDM model computed in the same position where $V_{\text {obs }}$ was measured, and $\mathbf{C}$ a diagonal matrix.

We sample the parameter space from uniform prior ranges with two Markov chains and tested the convergence of the fit with the Gelman-Rubin convergence criterion $(\mathcal{R}-1<0.1)$ (Gelman \& Rubin 1992). The fitting para- 
Table 1. High-resolution LSB galaxies. In this Table we show the characteristics of the 18 high-resolution LSB galaxies (de Blok et al. 2001) used in the present work. The columns read: (1) The name of the galaxy, (2) Morphology, (3) Absolute magnitude, (4) Maximum observational radius, (5) Maximum velocity of the rotation curve and (6) Distance to the galaxy.

\begin{tabular}{|c|c|c|c|c|c|}
\hline $\begin{array}{l}\text { Galaxy } \\
\text { Name } \\
\text { (1) }\end{array}$ & Morphology & $\begin{array}{c}M_{\mathrm{abs}} \\
(\mathrm{mag}) \\
(3)\end{array}$ & $\begin{array}{c}R_{\max } \\
(\mathrm{kpc}) \\
(4)\end{array}$ & $\begin{array}{c}V_{\max } \\
(\mathrm{km} / \mathrm{s}) \\
(5)\end{array}$ & $\begin{array}{c}D \\
(\mathrm{Mpc}) \\
(6)\end{array}$ \\
\hline ESO-LV 014-0040 & Spiral & -21.6 & 29.2 & 263 & 212 \\
\hline ESO-LV 084-0411 & Edge-on & -18.1 & 8.9 & 61 & 80 \\
\hline ESO-LV 120-0211 & Fuzzy Magellanic bar & -15.6 & 3.5 & 25 & 15 \\
\hline ESO-LV 187-0510 & Irregular spiral, flocculent & -16.5 & 3.0 & 40 & 18 \\
\hline ESO-LV 206-0140 & Spiral & -19.2 & 11.6 & 118 & 60 \\
\hline ESO-LV 302-0120 & Spiral, hint of bar? & -19.1 & 11.0 & 86 & 69 \\
\hline ESO-LV 305-0090 & Barred spiral & -17.3 & 4.8 & 54 & 11 \\
\hline ESO-LV 425-0180 & Spiral & -20.5 & 14.4 & 145 & 86 \\
\hline ESO-LV 488-0490 & Inclined Magellanic bar & -16.8 & 6.0 & 97 & 22 \\
\hline F730-V1 & Spiral & $\ldots$ & 11.9 & 145 & 144 \\
\hline UGC 4115 & Im & -12.4 & 1.0 & 40 & 3.2 \\
\hline UGC 11454 & Fuzzy spiral, small core & $-18.6^{a}$ & 11.9 & 152 & 91 \\
\hline UGC 11557 & SBdms & -20.0 & 6.2 & 95 & 22 \\
\hline UGC 11583 & dI & $-14.0^{a}$ & 1.5 & 36 & 5 \\
\hline UGC 11616 & $\mathrm{Sc}$ & $-20.3^{a}$ & 9.6 & 143 & 73 \\
\hline UGC 11648 & I & $-21.0^{a}$ & 12.7 & 145 & 48 \\
\hline UGC 11748 & Sbc & $-22.9^{a}$ & 21.0 & 242 & 73 \\
\hline UGC 11819 & $\mathrm{dG}$ & $-20.3^{a}$ & 11.7 & 153 & 60 \\
\hline
\end{tabular}

${ }^{a}$ Zwicky magnitude 17.

Table 2. Galaxies with photometric information. The same as Table 1 for the galaxies with photometric data.

\begin{tabular}{|c|c|c|c|c|c|}
\hline $\begin{array}{l}\text { Galaxy } \\
\text { Name } \\
(1)\end{array}$ & $\begin{array}{c}\text { Morphology } \\
\text { (2) }\end{array}$ & $\begin{array}{c}M_{\mathrm{abs}} \\
(\mathrm{mag}) \\
(3)\end{array}$ & $\begin{array}{c}R_{\max } \\
(\mathrm{kpc}) \\
(4)\end{array}$ & $\begin{array}{c}V_{\max } \\
(\mathrm{km} / \mathrm{s}) \\
(5)\end{array}$ & $\begin{array}{c}D \\
(\mathrm{Mpc}) \\
(6)\end{array}$ \\
\hline NGC $7814^{a}$ & Sabl & $-20.15^{d}$ & 5.03 & 218.9 & 14.40 \\
\hline NGC $6503^{a}$ & Scd & $-17.7^{e}$ & 3.04 & 116.3 & 6.26 \\
\hline NGC $3741^{a}$ & $\mathrm{Im}$ & $-13.13^{f}$ & 5.14 & 50.1 & 3.21 \\
\hline NGC $1003^{a}$ & $\mathrm{SA}(\mathrm{s}) \mathrm{cd}$ & -19.2 & 31.3 & 115 & 11.8 \\
\hline NGC $1560^{b}$ & Sd & -15.9 & 8.3 & 78 & 3.0 \\
\hline NGC $6946^{c}$ & SABcd & - & - & 224.3 & 10.1 \\
\hline
\end{tabular}

${ }^{a}$ Data from the SPARC database (Lelli et al. 2016).

${ }^{b}$ Data from de Blok et al. (2001).

${ }^{c}$ Data from McGaugh (2005).

${ }^{d}$ Monachesi et al. (2016).

${ }^{e}$ Koda et al. (2015).

${ }^{f}$ Dutta et al. (2009).

meters and $1 \sigma$ and $2 \sigma$ confidence levels (CL) are computed from the Markov chains with $30 \%$ as burn-in.

Simultaneously, we used the data analysis software ROOT (Brun \& Rademakers 1997), to minimize the $\chi^{2}$ errors to obtain the best fit from the observations:

$\chi_{\text {red }}^{2}=\frac{1}{N-N_{p}} \sum_{i=1}^{N}\left(\frac{V_{\text {obs }}\left(r_{i}\right)-V_{\text {model }}\left(r_{i}, \mathbf{p}\right)}{\sigma_{i}}\right)^{2}$,

where $N$ is the number of data points, $N_{p}$ the number of parameters and $\sigma_{i}$ the error in the measurement of $V_{\text {obs }}\left(r_{i}\right)$.

\section{RESULTS}

In this Section we show the results of the fits with the two SFDM models. In Subsection 4.1 we present the res- ults for the FDM model with the soliton+NFW density profile (4), separately for the high-resolution LSB galaxies (Subsection 4.1.1) and for the galaxies with photometric information (Subsection 4.1.3). In 4.1.2 we show the results of the combined analysis of the LSB galaxies to determine the single boson mass needed to fit all the rotation curves together. In Subsection 4.2 we show the results for the mSFDM model with the density profile (10), also separating the fits with the high-resolution LSB galaxies (Subsection 4.2.1) and the galaxies with photometric data (Subsection 4.2.2).

\subsection{Soliton+NFW fits}

\subsubsection{High-resolution LSB galaxies}

Table 3 shows the results for the 18 high-resolution LSB galaxies reported in de Blok et al. (2001), for the FDM model with the soliton+NFW density profile (4). As explained in Appendix A, we restricted the density profile asking for continuity of the function (4) at the transition radius $r_{\epsilon}$, as an abrupt transition between the soliton and NFW regions, as showed in the cosmological simulations (Schive et al. 014a,b; Schwabe et al. 2016; Mocz et al. 2017). With this restriction, we have four free parameters: the central density $\rho_{c}$, the soliton core radius $r_{c}$, the transition radius $r_{\epsilon}$, and the NFW characteristic radius $r_{s}$. For these quantities we report the $1 \sigma$ errors from the MCMC method used, also the $\chi_{\text {red }}^{2}$ errors for each galaxy. From $\rho_{c}$ and $r_{c}$ we obtained the boson mass $m_{\psi}$, and with the four free parameters the NFW density parameter $\rho_{s}$ can be computed. Additionally, 
we derive the virial radius $r_{200}$ and concentration parameter $c:=r_{200} / r_{s}{ }^{2}$ shown in the same Table.

From the best fit for each galaxy reported in Table 3, we notice that the ratio between the transition and core radii is in the interval $0.46 \leqslant r_{\epsilon} / r_{c} \leqslant 1.70$. This means that, in many cases, the soliton contribution is overlapped with the NFW halo in order to fit the observations. In all cases, the values of $r_{\epsilon}$ do not correspond to $r_{\epsilon}>3 r_{c}$, as found in the cosmological simulations (Schive et al. 014a). Thus, the soliton contribution is not as prominent as in the cosmological simulations, according to the best fits in the LSB galaxies.

For the 18 galaxies, the values for the boson mass are in the range $0.278 \leqslant m_{\psi} / 10^{-23} \mathrm{eV} \leqslant 27.0$, and core radius within $0.326 \leqslant r_{c} / \mathrm{kpc} \leqslant 8.96$. The wide range in core radii is consistent with the variety of galaxy sizes. It most be noticed that the half of the galaxies in the sample have resulting boson masses $m_{\psi}<10^{-23} \mathrm{eV}$, in disagreement with the strongest cosmological constraints (Bozek et al. 2015; Sarkar et al. 2016). For those rotation curves, we tried to restrict the boson mass to be at least $m_{\psi}=10^{-23} \mathrm{eV}$, but it was not possible to fit the data. In these cases, the FDM model is in tension with the LSB observations. And as we show in Subsection 4.1.3, the low masses $m_{\psi}<10^{-23} \mathrm{eV}$ persist in the analysis of the NGC galaxies with photometric data.

Fig. 1 shows the $1 \sigma$ and $2 \sigma$ contours for the parameters $m_{\psi}$ and $r_{c}$ from the MCMC analysis, and the transition radius $r_{\epsilon}$ is the scatter plot. From this figure, it can be noticed the degeneracy in the boson mass $m_{\psi}$ between the galaxies, appearing to be inconsistent with each other. However, the boson mass must be a constant of the model, changing only its core radius to fit the observations. In the next Subsection we report the results of the combined analysis for the 18 LSB galaxies, keeping the boson mass as constant.

\subsubsection{Combined analysis for the LSB galaxies}

Taking into account that the mass of the ultra-light SF particle in the SFDM model must be a constant, in this Subsection we report the results of a combined analysis fitting all the LSB galaxies with a single mass $m_{\psi}$, leaving the other parameters free for each galaxy. This analysis was performed minimizing the $\chi^{2}$ errors for the whole data of the 18 LSB galaxies to obtain the best-fit mass from the observations. Notice that we only include the LSB galaxies, as a sample spanning a wide range in sizes and morphological types, to lead with DM-only fits and not with the baryons. The results are shown in Table 4, where we report the free parameters $\rho_{c}, r_{c}, r_{\epsilon}$ and $r_{s}$, the resulting constant boson mass $m_{\psi}$ from the analysis, the ratio $r_{\epsilon} / r_{c}$, and the NFW density $\rho_{s}$, virial radius $r_{200}$ and concentration parameter $c$.

The best-fit mass obtained in the combined analysis is $m_{\psi}=0.554 \times 10^{-23} \mathrm{eV}$, with a total reduced error $\chi_{\text {red }}^{2}=$ 1.208. Unfortunately, the computation time of the combined analysis was too expensive and we were not able to obtain

${ }^{2} r_{200}$ is the radius where the density is 200 times the critical density of the Universe, and defines the halo radius. In the CDM framework, concentrations are strongly correlated with the halo formation epoch, and must be in agreement with the $N$-body simulations. the confidence level for the FDM boson mass. However, we believe that it is not too large given the errors obtained in the individual analysis of these galaxies (see Table 3 ) and the value of the combined $\chi_{\text {red }}^{2}$ we obtained here. Thus, it is possible to fit all the galaxies together but with a very small mass that does not meet the cosmological constraint imposed to the FDM model (Bozek et al. 2015; Sarkar et al. 2016). If we impose the condition $m_{\psi}>10^{-23} \mathrm{eV}$ into the fitting method, it is not possible to fit all the rotation curves in a combined analysis. These results set a tension between the FDM model and the observations.

In this case, the soliton core radii are in the interval $1.57 \leqslant r_{c} / \mathrm{kpc} \leqslant 5.82$ and ratio between the transition and core radii within $0.30 \leqslant r_{\epsilon} / r_{c} \leqslant 2.3$. Again, the best fits do not correspond to $r_{\epsilon}>3 r_{c}$, as expected in the cosmological simulations (Schive et al. 014a). In both cases, the individual and combined analysis, the soliton contribution required to fit the observations is not as prominent as in the simulations.

Fig. 2 shows the resulting rotation curves for each galaxy with the fitting parameters shown in Table 4. The transition radius $r_{\epsilon}$ is the vertical dashed line shown for all the galaxies. Remarkably, with a constant boson mass the fits are good for almost all the galaxies, given the large observational errors for some galaxies in the sample, except for ESO 425-0180, UGC 11583, 11648 and 11748, and some regions in ESO 206-0140.

\subsubsection{NGC galaxies with photometric data}

In Table 5 we show the fitting parameters for the three representative galaxies in McGaugh et al. (2016) (NGC 7814, 6503, 3741) from the SPARC database (Lelli et al. 2016) and the three sample galaxies analyzed in Robles \& Matos (2013b) (NGC 1003, data from SPARC; NGC 1560, data from de Blok et al. (2001); NGC 6946, data from McGaugh (2005)), for the FDM model with the soliton+NFW density profile. In the top panel we show the results for the galaxies without the photometric information, and on the bottom panel the results including the disk, gas and/or bulge contribution, to notice the effect of the baryons on the rotation curves. In each case, we report the fitting parameters $\rho_{c}$, $r_{c}, r_{\epsilon}$ and $r_{s}$, with the resulting boson mass $m_{\psi}$, the ratio $r_{\epsilon} / r_{c}$, the virial radius $r_{200}$, concentration parameter $c$, the $\chi_{\text {red }}^{2}$ error and $\pm 1 \sigma$ uncertainties from the MCMC method.

In Fig. 3 we show the plots for the corresponding fitting parameters presented in Table 5, for the galaxies without and with photometric data; the vertical lines show the corresponding transition radius $r_{\epsilon}$ for each galaxy. Fig. 1 shows the $1 \sigma$ and $2 \sigma$ contours for $m_{\psi}$ and $r_{c}$ for the NGC galaxies with photometric data, where $r_{\epsilon}$ is the scatter plot.

We notice that, in general, the fits are improved with the baryons inclusion, especially at the innermost regions. For NGC 7814, the bulge-dominated galaxy, both fits (with and without photometric data) are very good; in this case the soliton is capable to reproduce the bulge behavior. For NGC 6503 and NGC 6946, both disk-dominated galaxies, the innermost regions are reproduced once the baryons are included. We have the same for NGC 1003, but as can be noticed, even when the fit is better, the soliton+NFW profile cannot explain the oscillations from the high-resolution observations; in this case the mSFDM profile is relevant (see Robles \& Matos 2013b, and Subsection 4.2). Finally, for 

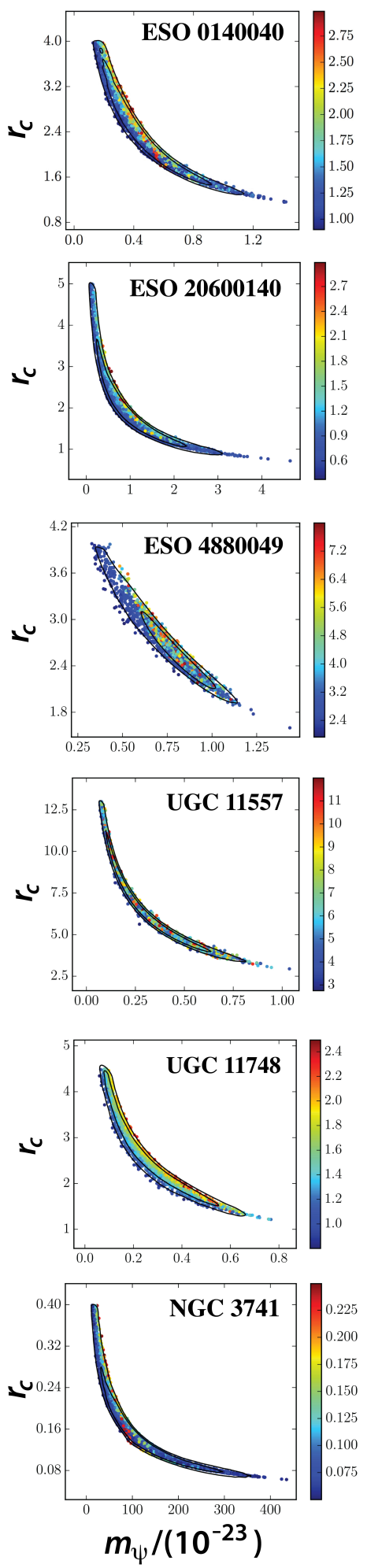
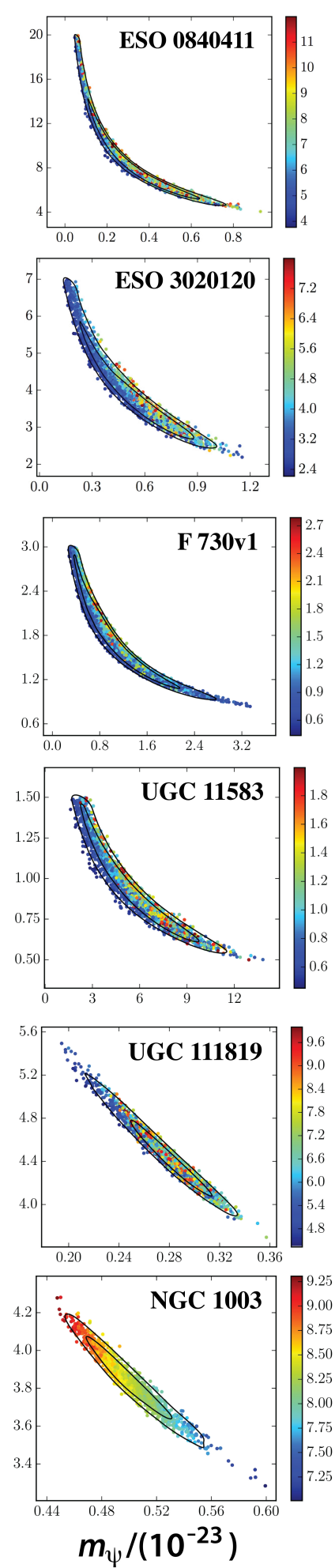
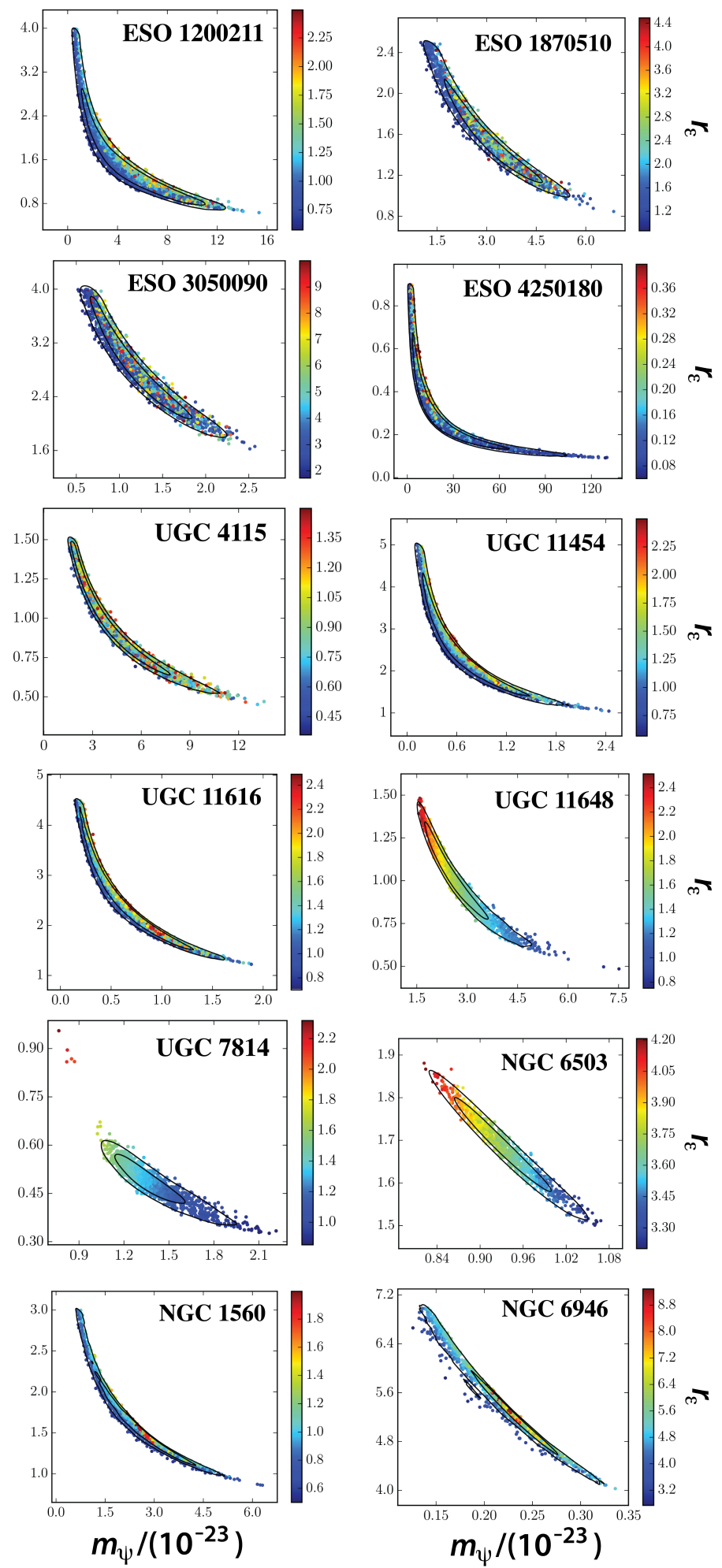

Figure 1. Posterior distributions for the parameters $m_{\psi}\left(10^{-23} \mathrm{eV}\right)$ and $r_{c}(\mathrm{kpc})$, with the transition radius $r_{\epsilon}(\mathrm{kpc})$ as the scatter plot, for the high-resolution LSB and NGC galaxies in the FDM model. The contours are the $1 \sigma$ and $2 \sigma$ confidence regions. The plots were obtained with the GetDist 0.2.6 Package. 

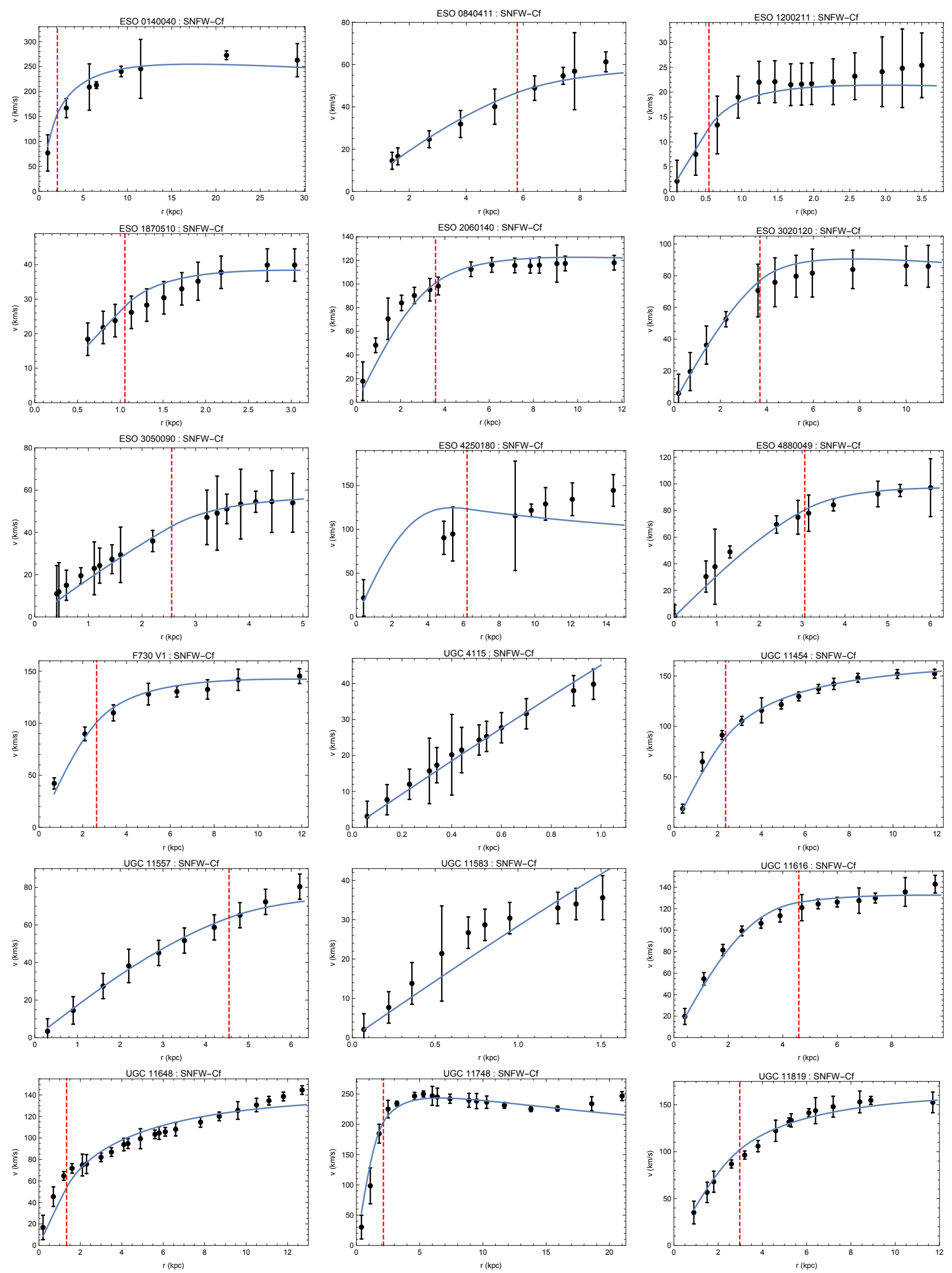

Figure 2. Best fits from the combined analysis for the 18 high-resolution LSB galaxies, in the FDM model using the soliton+NFW profile. The corresponding fitting parameters are shown in Table 4 , for a constant boson mass $m_{\psi}=0.554 \times 10^{-23} \mathrm{eV}$. The vertical lines correspond to the transition radii $r_{\epsilon}$. In all cases, the soliton contribution is important in order to fit the whole rotation curve. 
Table 3. Soliton+NFW density profile in high-resolution LSB galaxies. In this Table we show the resulting fitting parameters $\rho_{c}, r_{c}, r_{\epsilon}$ and $r_{s}$ for the FDM model with the soliton+NFW density profile (4), and the resulting boson mass $m_{\psi}$, all the quantities $\pm 1 \sigma$ errors from the MCMC method used. We also show the resulting ratio $r_{\epsilon} / r_{c}$, the $r_{200}$ radius from the NFW halo, concentration parameter $c$ and $\chi_{\text {red }}^{2}$ errors for the 18 high-resolution LSB galaxies in de Blok et al. (2001).

\begin{tabular}{|c|c|c|c|c|c|c|c|c|c|}
\hline Galaxy & $\begin{array}{c}\rho_{c} \\
\left(10^{-2} M_{\odot} / \mathrm{pc}^{3}\right)\end{array}$ & $\begin{array}{c}r_{c} \\
(\mathrm{kpc})\end{array}$ & $\begin{array}{c}r_{\epsilon} \\
(\mathrm{kpc})\end{array}$ & $r_{\epsilon} / r_{c}$ & $\begin{array}{c}m_{\psi} \\
\left(10^{-23} \mathrm{eV}\right)\end{array}$ & $\begin{array}{c}r_{s} \\
(\mathrm{kpc})\end{array}$ & $\begin{array}{l}r_{200} \\
(\mathrm{kpc})\end{array}$ & $c$ & $\chi_{\mathrm{red}}^{2}$ \\
\hline ESO 014-0040 & $36.8 \pm 8.9$ & $2.38_{-0.94}^{+0.42}$ & $1.59_{-0.68}^{+0.21}$ & 0.67 & $0.50_{-0.32}^{+0.13}$ & $15.3_{-3.8}^{+2.6}$ & 287 & 18.8 & 6.22 \\
\hline ESO 084-0411 & $0.483_{-0.11}^{+0.068}$ & $8.96_{-3.9}^{+0.94}$ & $7.7_{-2.7}^{+1.30}$ & 0.86 & $0.33_{-0.25}^{+0.12}$ & $1.56_{-1.5}^{+0.29} \times 10^{-5}$ & 123 & $7.90 \times 10^{6}$ & 0.878 \\
\hline ESO $120-0211$ & $2.70_{-1.2}^{+0.60}$ & $1.59_{-0.83}^{+0.18}$ & $1.22_{-0.47}^{+0.15}$ & 0.77 & $5.2_{-4.5}^{+1.25}$ & $1.47_{-1.5}^{+0.19} \times 10^{-3}$ & 30.3 & $2.06 \times 10^{4}$ & 1.03 \\
\hline ESO 187-0510 & $3.95_{-1.0}^{+0.5}$ & $1.59_{-0.42}^{+0.21}$ & $\begin{array}{r}2.17_{-0.99}^{+0.29} \\
+0.99\end{array}$ & 1.36 & $\begin{array}{l}-4.5 \\
3.06 \pm 0.97\end{array}$ & $1.67_{-1.7}^{+0.44} \times 10^{-2}$ & 42.6 & $2.55 \times 10^{3}$ & 0.44 \\
\hline ESO 206-0140 & $20.4_{-5.7}^{+4.5}$ & $1.98_{-0.93}^{+0.31}$ & $1.10_{-0.46}^{+0.21}$ & 0.56 & $1.09_{-0.86}^{+0.31}$ & $6.0_{-1.8}^{+1.3}$ & 123 & 20.4 & 3.13 \\
\hline ESO 302-0120 & $3.55_{-0.82}^{+0.57}$ & $3.98_{-1.3}^{+0.39}$ & $4.25_{-1.5}^{+0.40}$ & 1.07 & $0.54_{-0.32}^{+0.23}$ & $1.74_{-1.7}^{+0.48} \times 10^{-3}$ & 106 & $6.09 \times 10^{4}$ & 0.58 \\
\hline ESO 305-0090 & $2.38_{-0.62}^{+0.35}$ & $2.81_{-0.68}^{+0.42}$ & $4.34_{-2.3}^{+0.62}$ & 1.54 & $1.24_{-0.49}^{+0.22}$ & $7.6_{-7.5}^{+1.8} \times 10^{-3}$ & 68.1 & $8.96 \times 10^{3}$ & 0.17 \\
\hline ESO $425-0180$ & $106_{-27.0}^{+51.0}$ & $0.326_{-0.22}^{+0.058}$ & $0.151_{-0.089}^{+0.021}$ & 0.46 & $27.0_{-30.0}^{+6.09}$ & $21_{-10}^{+7}$ & 205 & 9.78 & 5.25 \\
\hline ESO 488-0490 & $7.15_{-1.4}^{+0.96}$ & $2.66_{-0.45}^{+0.22}$ & $4.13_{-1.8}^{+0.559}$ & 1.55 & $0.77_{-0.12}^{+0.10}$ & $1.08_{-1.1}^{+0.37} \times 10^{-4}$ & 108 & $1.00 \times 10^{6}$ & 0.83 \\
\hline F730-V1 & $23.6_{-6.7}^{+4.4}$ & $1.75_{-0.67}^{+0.31}$ & $1.16_{-0.55}^{+0.19}$ & 0.66 & $1.16_{-0.77}^{+0.27}$ & $9.3_{-3.0}^{+2.1}$ & 160 & 17.2 & 5.72 \\
\hline UGC 4115 & $15.5_{-3.3}^{+1.8}$ & $0.96_{-0.34}^{+0.18}$ & $0.94_{-0.34}^{+0.16}$ & 0.98 & $4.62_{-2.8}^{+0.93}$ & $1.90_{-0.91}^{+0.48} \times 10^{-6}$ & 46.7 & $2.46 \times 10^{7}$ & 0.084 \\
\hline UGC 11454 & $17.3_{-5.1}^{+3.0}$ & $2.56_{-1.2}^{+0.47}$ & $1.29_{-0.51}^{+0.25}$ & 0.50 & $0.72_{-0.55}^{+0.21}$ & $12.6_{-2.8}^{+2.0}$ & 185 & 14.6 & 9.09 \\
\hline UGC 11557 & $1.48_{-0.33}^{+0.17}$ & $6.7_{-2.9}^{+1.0}$ & $6.7_{-2.9}^{+1.11}$ & 1.00 & $0.335_{-0.25}^{+0.089}$ & $1.88_{-0.89}^{+0.10} \times 10^{-3}$ & 129 & $6.88 \times 10^{4}$ & 0.389 \\
\hline UGC 11583 & $10.2_{-3.2}^{+1.6}$ & $0.95_{-0.32}^{+0.15}$ & $1.04_{-0.44}^{+0.14}$ & 1.09 & $5.6_{-3.1}^{+0.5}$ & $2.27_{-1.3}^{+0.89} \times 10^{-6}$ & 42.3 & $1.86 \times 10^{7}$ & 0.617 \\
\hline UGC 11616 & $16.6_{-3.8}^{+2.3}$ & $2.51_{-1.0}^{+0.32}$ & $1.48_{-0.50}^{+0.24}$ & 0.59 & $0.69_{-0.49}^{+0.20}$ & $7.2_{-2.5}^{+1.7}$ & 142 & 19.8 & 3.49 \\
\hline UGC 11648 & $29.5_{-10.0}^{+3.6}$ & $1.01 \pm 0.19$ & $1.72 \pm 0.31$ & 1.70 & $2.73_{-0.88}^{+0.37}$ & $54_{-20}^{+10.5}$ & 285 & 5.28 & 1.08 \\
\hline UGC 11748 & $86.0_{-25.0}^{+12.0}$ & $2.63_{-1.1}^{+0.52}$ & $1.58_{-0.38}^{+0.27}$ & 0.60 & $0.283_{-0.20}^{+0.085}$ & $2.24 \pm 0.32$ & 170 & 76.0 & 11.6 \\
\hline UGC 11819 & $6.35_{-0.44}^{+0.40}$ & $4.46_{-0.28}^{+0.19}$ & $6.97_{-1.8}^{+0.84}$ & 1.56 & $0.278_{-0.019}^{+0.025}$ & $1.72_{-0.71}^{+0.25} \times 10^{-4}$ & 175 & $1.01 \times 10^{6}$ & 0.408 \\
\hline
\end{tabular}

Table 4. Soliton+NFW density profile - Combined analysis in high-resolution LSB galaxies. In this Table we show the resulting fitting parameters $\rho_{c}, r_{c}, r_{\epsilon}$ and $r_{s}$ for the FDM model with the soliton+NFW density profile (4). The resulting boson mass is $m_{\psi}=$ $0.554 \times 10^{-23} \mathrm{eV}$. The combined analysis was performed minimizing the $\chi^{2}$ errors for the whole sample, and we obtained $\chi_{\text {red }}^{2}=1.208$. We also show the resulting NFW density parameter $\rho_{s}$, the ratio $r_{\epsilon} / r_{c}$, the $r_{200}$ radius from the NFW halo, concentration parameter $c$ and total DM mass $M_{200}=M_{\mathrm{FDM}}\left(r_{200}\right)$, for the 18 high-resolution LSB galaxies in de Blok et al. (2001).

\begin{tabular}{|c|c|c|c|c|c|c|c|c|c|c|}
\hline Galaxy & $\begin{array}{c}\rho_{c} \\
\left(10^{-2} M_{\odot} / \mathrm{pc}^{3}\right)\end{array}$ & $\begin{array}{c}r_{c} \\
(\mathrm{kpc})\end{array}$ & $\begin{array}{c}r_{\epsilon} \\
(\mathrm{kpc})\end{array}$ & $r_{\epsilon} / r_{c}$ & $\begin{array}{c}m_{\psi} \\
\left(10^{-23} \mathrm{eV}\right)\end{array}$ & $\begin{array}{c}r_{s} \\
(\mathrm{kpc})\end{array}$ & $\begin{array}{c}\rho_{s} \\
\left(10^{-2} M_{\odot} / \mathrm{pc}^{3}\right)\end{array}$ & $c$ & $\begin{array}{l}r_{200} \\
(\mathrm{kpc})\end{array}$ & $\begin{array}{c}M_{200} \\
\left(10^{11} M_{\odot}\right)\end{array}$ \\
\hline ESO 014-0040 & 50.2 & 1.87 & 2.09 & 1.1 & 0.554 & 7.87 & 9.07 & 29.3 & 231 & 13.5 \\
\hline ESO 084-0411 & 0.542 & 5.82 & 5.79 & 1.0 & 0.554 & 4.42 & 1.90 & 16.0 & 70.6 & 0.36 \\
\hline ESO 120-0211 & 3.09 & 3.76 & 0.54 & 0.15 & 0.554 & 1.18 & 3.02 & 19.2 & 22.5 & 0.010 \\
\hline ESO 187-0510 & 4.04 & 3.52 & 1.06 & 0.30 & 0.554 & 0.73 & 33.0 & 47.8 & 34.7 & 0.040 \\
\hline ESO 206-0140 & 7.78 & 2.99 & 3.58 & 1.2 & 0.554 & 3.28 & 13.9 & 34.5 & 113 & 1.55 \\
\hline ESO 302-0120 & 3.67 & 3.60 & 3.70 & 1.0 & 0.554 & 1.20 & 90.8 & 69.6 & 83.5 & 0.57 \\
\hline ESO 305-0090 & 1.82 & 4.30 & 2.55 & 0.59 & 0.554 & 1.70 & 13.4 & 34.0 & 57.6 & 0.20 \\
\hline ESO $425-0180$ & 10.9 & 2.74 & 6.21 & 2.3 & 0.554 & 2.13 & 22.9 & 41.6 & 88.7 & 0.82 \\
\hline ESO 488-0490 & 5.52 & 3.26 & 3.06 & 0.94 & 0.554 & 1.13 & 109 & 74.6 & 84.6 & 0.60 \\
\hline F730-V1 & 12.08 & 2.68 & 2.64 & 0.99 & 0.554 & 4.62 & 8.63 & 28.8 & 133 & 2.57 \\
\hline UGC 4115 & 12.0 & 2.68 & 1.26 & 0.47 & 0.554 & 1.53 & 28.0 & 44.9 & 68.8 & 0.35 \\
\hline UGC 11454 & 10.7 & 2.76 & 2.36 & 0.86 & 0.554 & 9.31 & 2.54 & 17.9 & 167 & 5.12 \\
\hline UGC 11557 & 1.70 & 4.37 & 4.55 & 1.0 & 0.554 & 2.26 & 14.6 & 35.1 & 79.4 & 0.50 \\
\hline UGC 11583 & 4.65 & 3.40 & 1.77 & 0.52 & 0.554 & 0.86 & 73.2 & 64.3 & 55.3 & 0.17 \\
\hline UGC 11616 & 11.8 & 2.69 & 4.59 & 1.7 & 0.554 & 4.21 & 8.60 & 28.7 & 121 & 1.95 \\
\hline UGC 11648 & 9.86 & 2.82 & 1.34 & 0.48 & 0.554 & 11.5 & 1.21 & 13.4 & 154 & 4.02 \\
\hline UGC 11748 & 102 & 1.57 & 2.13 & 1.4 & 0.554 & 2.60 & 80.3 & 66.5 & 173 & 5.65 \\
\hline UGC 11819 & 10.3 & 2.78 & 2.98 & 1.1 & 0.554 & 8.98 & 2.75 & 18.5 & 166 & 5.04 \\
\hline
\end{tabular}

NGC 3741 and 1560, the total velocities overestimate the observational points for the outer radii, even once the baryons have been included.

The central soliton densities are larger in the DM-only analysis since the soliton must fit the rotation curves at the innermost radii where the baryons might contribute more, especially in NGC 7814. As can be easily seen from Fig. 3, we notice that in all cases the transition radii increase once the baryons have been included, consistent with those densities. The same occurs for the soliton core radii (except for NGC 6946). Therefore, the soliton is less concentrated than in the DM-only study.

Finally, the boson masses for the DM+baryons analysis are in the range $0.212 \leqslant m_{\psi} / 10^{-23} \mathrm{eV} \leqslant 12.1$, and core radii within $0.759 \leqslant r_{c} / \mathrm{kpc} \leqslant 5.47$. We notice again that 4 of 6 galaxies have corresponding boson masses $m_{\psi}<10^{-23} \mathrm{eV}$, 
Table 5. Soliton+NFW density profile in NGC galaxies with photometric data. The top panel shows the DM-only fits and the bottom panel the DM+baryons analysis, both for the same galaxies with high-resolution photometric information. Both panels show the fitting parameters $\rho_{c}, r_{c}, r_{\epsilon}$ and $r_{s}$, for the FDM model with the soliton+NFW density profile (4), and the resulting boson mass $m_{\psi}$; all the quantities $\pm 1 \sigma$ errors from the MCMC method used. We also show the resulting ratio $r_{\epsilon} / r_{c}$, the $r_{200}$ radius from the NFW halo, concentration parameter $c$ and $\chi_{\text {red }}^{2}$ errors.

\begin{tabular}{|c|c|c|c|c|c|c|c|c|c|}
\hline \multicolumn{10}{|c|}{ DM-only fits } \\
\hline Galaxy & $\begin{array}{c}\rho_{c} \\
\left(10^{-2} M_{\odot} / \mathrm{pc}^{3}\right)\end{array}$ & $\begin{array}{c}r_{c} \\
(\mathrm{kpc})\end{array}$ & $\begin{array}{c}r_{\epsilon} \\
(\mathrm{kpc})\end{array}$ & $r_{\epsilon} / r_{c}$ & $\begin{array}{c}m_{\psi} \\
\left(10^{-23} \mathrm{eV}\right)\end{array}$ & $\begin{array}{c}r_{s} \\
(\mathrm{kpc})\end{array}$ & $\begin{array}{l}r_{200} \\
(\mathrm{kpc})\end{array}$ & $c$ & $\chi_{\mathrm{red}}^{2}$ \\
\hline NGC 7814 & $1910_{-510}^{+300}$ & $0.485 \pm 0.057$ & $1.29_{-0.14}^{+0.16}$ & 2.7 & $1.39_{-0.20}^{+0.11}$ & $5.17_{-0.93}^{+0.67}$ & 168 & 32.4 & 2.48 \\
\hline NGC 6503 & $27.3_{-2.4}^{+2.0}$ & $1.686 \pm 0.072$ & $3.70 \pm 0.18$ & 2.2 & $0.933_{-0.049}^{+0.040}$ & $9.73_{-1.0}^{+0.93}$ & 116 & 11.9 & 1.12 \\
\hline NGC 3741 & $42.0 \pm 12.0$ & $0.163_{-0.091}^{+0.023}$ & $0.0962_{-0.047}^{+0.0092}$ & 0.59 & $129_{-100}^{+40}$ & $15.7_{-4.4}^{+2.5}$ & 93.5 & 5.96 & 8.4 \\
\hline NGC 1003 & $3.51_{-0.27}^{+0.23}$ & $3.84 \pm 0.15$ & $8.36_{-0.30}^{+0.36}$ & 2.2 & $0.501_{-0.023}^{+0.018}$ & $128_{-50}^{+3.4}$ & 223 & 1.74 & 1.29 \\
\hline NGC 1560 & $5.67_{-0.90}^{+0.54}$ & $1.64_{-0.52}^{+0.16}$ & $1.07_{-0.33}^{+0.13}$ & 0.65 & $2.5 \pm 1.0$ & $12.7_{-1.7}^{+1.3}$ & 8.06 & 102 & 2.98 \\
\hline NGC 6946 & $4.87_{-0.46}^{+0.31}$ & $5.34_{-0.57}^{+0.32}$ & $\begin{array}{c}6.1_{-1.6}^{+1.3 .0} \\
\text { +1.3. }\end{array}$ & 1.1 & $0.225_{-0.024}^{+0.036}$ & $\begin{array}{l}5.7_{-1.5}^{+1.1} \cdot{ }^{7} \\
\end{array}$ & 157 & 27.5 & 15.4 \\
\hline \multicolumn{10}{|c|}{ DM+baryons fits } \\
\hline NGC 7814 & $4.85_{-0.68}^{+0.46}$ & $5.47 \pm 0.40$ & $9.0 \pm 1.0$ & 1.65 & $0.212_{-0.023}^{+0.016}$ & $21_{-10}^{+6}$ & 214 & 10.2 & 0.668 \\
\hline NGC 6503 & $4.07_{-0.40}^{+0.32}$ & $3.31 \pm 0.20$ & $5.59 \pm 0.51$ & 1.69 & $0.628_{-0.056}^{+0.040}$ & $11.5_{-2.0}^{+1.5}$ & 114 & 9.95 & 1.18 \\
\hline NGC 3741 & $5.7_{-1.6}^{+1.40}$ & $0.759_{-0.28}^{+0.098}$ & $0.615_{-0.22}^{+0.069}$ & 0.81 & $12.1_{-6.7}^{+3.956}$ & $23_{-10}^{+4.0}$ & 101 & 4.39 & 2.9 \\
\hline NGC 1003 & $1.80_{-0.19}^{+0.14}$ & $4.52 \pm 0.24$ & $8.76_{-0.43}^{+0.50}$ & 1.94 & $0.507_{-0.035}^{+0.7}$ & $159_{-80}^{+40}$ & 232 & 1.46 & 1.33 \\
\hline NGC 1560 & $\begin{array}{r}4.22_{-0.79}^{+0.46} \\
\text { +. }\end{array}$ & $1.74_{-0.59}^{+0.22}$ & $1.10_{-0.38}^{+0.14}$ & 0.63 & $2.65_{-1.6}^{+0.85}$ & $13.7_{-2.4}^{+1.8}$ & 96.0 & 7.01 & 2.42 \\
\hline NGC 6946 & $2.99_{-0.55}^{+0.21}$ & $\begin{array}{l}4.56_{-0.43}^{+0.59} \\
\end{array}$ & $8.0 \pm 1.0$ & 1.75 & $0.395_{-0.077}^{+0.039}$ & $71_{-30}^{+20}$ & 242 & 3.40 & 2.03 \\
\hline
\end{tabular}

a result that does not meet the cosmological constraints (Bozek et al. 2015; Sarkar et al. 2016), setting a possible tension between the FDM model and the rotation curves of these galaxies.

\subsection{Multistate SFDM fits}

Here we present the results for the multistate SFDM model, with the density profile (10). The reported combinations of excited states are the ones that best reproduce the data.

The preferred state we are looking for is the ground state $(j=1)$, and combinations including it, since we expect the galaxy halos in the SFDM scenario were formed from a BEC with this initial condition. As the halos evolve, a realistic approximation describing the bosons in such configurations is to expect that those particles reach higher energy levels due to galaxy and galaxy clusters formation processes and their interaction with the baryonic matter. Within this hypothesis, it is reasonable to expect lower excitation states, since they are more easily attainable than the higher levels, and for the rotation curves analyzed here, radii corresponding to lower excitation states are enough to fit the observations. Therefore we preferred lower states to restrict the freedom in $j$. Further numerical simulations are needed to investigate the distribution of excitation states in diverse environments and under different initial conditions, and their comparison with observations.

Now, it is a well-known result that it is not possible to reproduce the whole rotation curves of large galaxies with the ground state only, since the corresponding velocity profile decreases very quickly after its maximum value (Boehmer \& Harko 2007; Robles \& Matos 2012; Guzmán \& Lora-Clavijo 2015). As shown below, it was possible to fit only some LSB galaxies with the single ground state $j=1$ and none of the NGC galaxies. Furthermore, it is important to note that in some cases, the resulting configurations do not display the ground state as a dominant component. This might be the result of different formation histories for each galaxy, including interactions with the baryonic matter resulting in diverse multistate configurations.

\subsubsection{High-resolution LSB galaxies}

In Table 6 we show the results for 11 of the 18 high-resolution LSB galaxies from de Blok et al. (2001), whose rotation curves can be reproduced with one state only. From them, only 5 can be fitted with the ground state $j=1$, and the other ones with an excited state $j>1$. We report the fitting parameters $j, R$ and the central density for the $j$-th state, $\rho_{0}^{j}$, all the quantities $\pm 1 \sigma$ errors from the MCMC method used. We report also the corresponding mSFDM mass $M_{j}=M_{\operatorname{mSFDM}}(R)$ and $\chi_{\text {red }}^{2}$ errors for each galaxy. In Fig. 4 we show the corresponding rotation curves for these galaxies, showing that the fits are well inside the observational errors.

In Table 7 we show the results for the remaining 7 LSB galaxies, reproduced with two excited states $i, j$. We report the fitting parameters, $\rho_{0}^{i}$ and $\rho_{0}^{j}$ (the central densities for the states $i$ and $j$ ) and the halo radius $R$, all the quantities $\pm 1 \sigma$ errors. We also computed the mass in the highest energy level $M_{j}$ and in the ground state $M_{1}$ (for the galaxies displaying $j=1$ ), as well as the mass ratio $\eta=M_{j} / M_{1}$. Ureña-López \& Bernal (2010) showed it is possible to have stable multistate configurations of the Schrödinger-Poisson system for values of $\eta=M_{j} / M_{1} \lesssim 1.3$, i.e. for this values in the simulations, the multistate halos do not decay to the ground state. Therefore, from our results, we might argue that the final configurations are stable where the ground state appears as a dominant component. Further numerical simulations are needed to test the validity of those results in the context of the mSFDM analytical model.

Additionally, there are 4 galaxies where the lowest dom- 


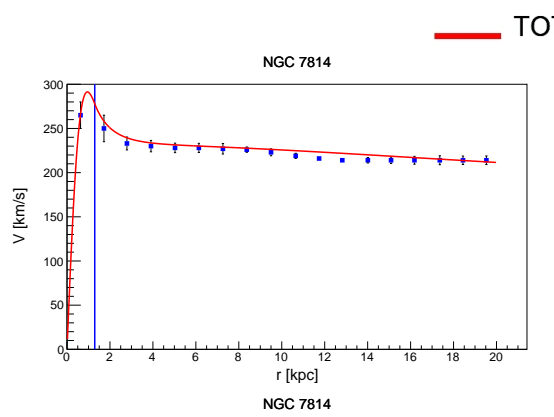

TOTAL RC $=\ldots$ STARS

BULGE

GAS FDM
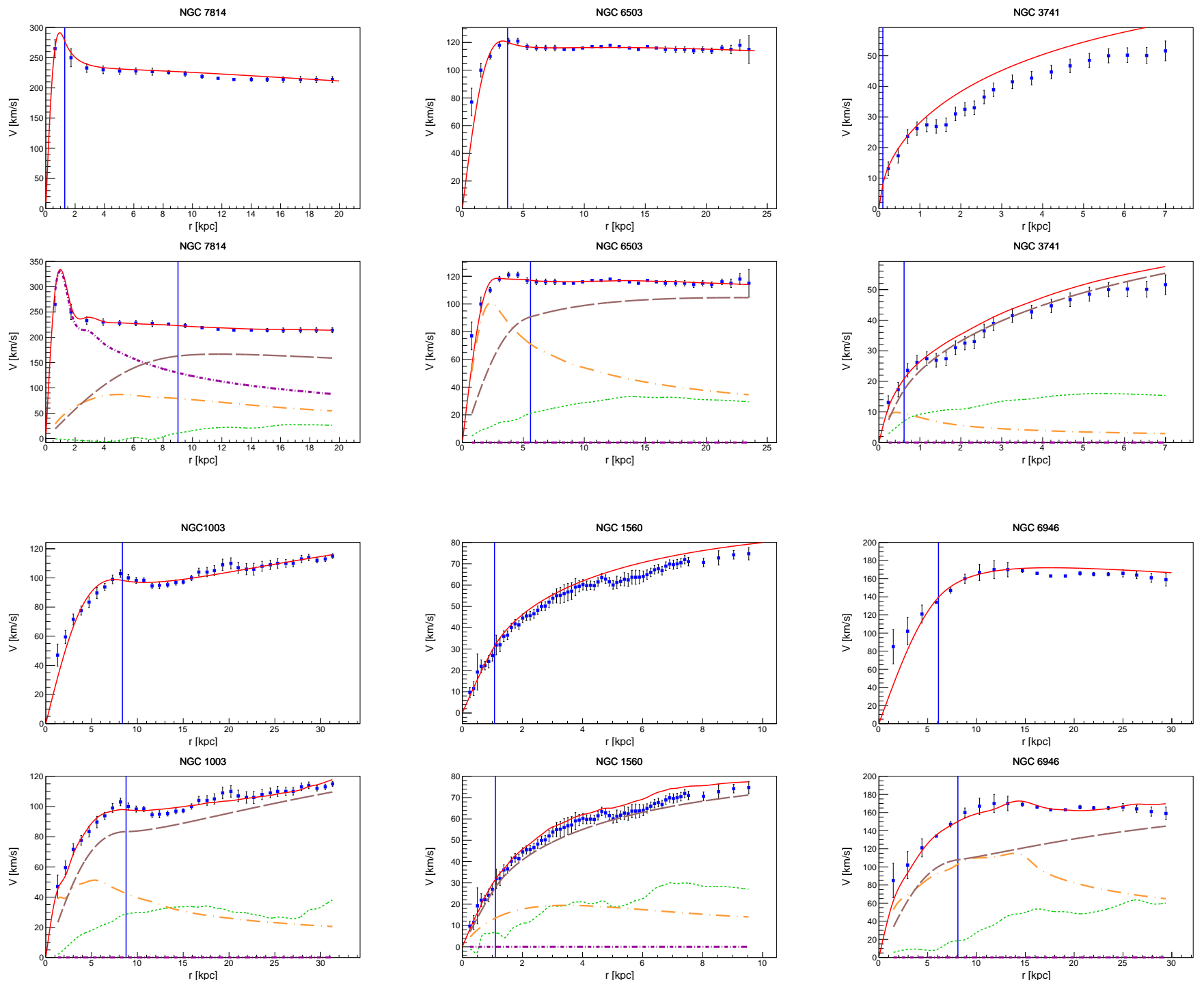

Figure 3. Best fits in the FDM model for the soliton+NFW profile for the NGC galaxies with photometric data. The fitting parameters are shown in Table 5. We compare the results without and taking into account the baryonic information. The vertical lines are the corresponding transition radii $r_{\epsilon}$ from the MCMC method. For all the galaxies, the soliton contribution is less concentrated at the inner radii once the baryonic data is taken into account.

inant state is $i=2$ or even $i=6$; in these systems, the total distribution in different excitation levels must be the final product of galaxy formation processes and interactions with the baryonic matter. Again, numerical simulations are needed to investigate the stability of such multistate configurations without a dominant ground state and the influence of the baryonic matter in the mSFDM halo. With the halos formed by two excitation states, we found a very good agreement with the observational velocities for the galaxies, except for UGC 11748. For this galaxy the fit might be improved by introducing a third excited state. Fig. 5 shows the resulting best-fit rotation curves for the parameters reported in Table 7.

\subsubsection{NGC galaxies with photometric data}

In Table 8 we present the results for the multistate SFDM model, for the 3 representative SPARC galaxies in McGaugh et al. (2016) (NGC 7814, 6503, 3741) and the 3 sample galaxies analyzed in Robles \& Matos (2013b) (NGC 1003, data from SPARC; NGC 1560, data from de Blok et al. (2001); NGC 6946, data from McGaugh (2005)). We present separately the DM-only fits and the DM+baryons fits including the baryonic data to reproduce the rotation curves. For these galaxies, it was not possible to fit the data with one state only, and we report the two states-combinations. For the halos formed with two states we report: $\rho_{0}^{i}, \rho_{0}^{j}$ and $R$, all $\pm 1 \sigma$ errors from the MCMC method. We also report the total masses $M_{i}$ and $M_{j}$, and the resulting mass ratios $\eta=M_{j} / M_{1}$ (where the ground state appears as a dominant component). For the DM+baryons fits we obtained 4 galax- 
Table 6. Multistate SFDM with one excited state in high-resolution LSB galaxies. In this Table we show the fitting parameters $R$ and $\rho_{0}^{j}$ for one excited state $j, \pm 1 \sigma$ errors from the MCMC method used, in the multistate SFDM model (10) for 11 high-resolution LSB galaxies in de Blok et al. (2001). We also report the resulting DM mass $M_{j}$ and $\chi_{\text {red }}^{2}$ errors from the fitting method.

\begin{tabular}{lccccc}
\hline Galaxy & $j$ & $\begin{array}{c}R \\
(\mathrm{kpc})\end{array}$ & $\begin{array}{c}\rho_{0}^{j} \\
\left(10^{-2} M_{\odot} / \mathrm{pc}^{3}\right)\end{array}$ & $\begin{array}{c}M_{j} \\
\left(10^{10} M_{\odot}\right)\end{array}$ & $\chi_{\text {red }}^{2}$ \\
\hline ESO 084-0411 & 1 & $18.0_{-5.4}^{+2.1}$ & $0.461_{-0.10}^{+0.058}$ & 0.836 & 0.347 \\
ESO 120-0211 & 3 & $8.4_{-2.1}^{+1.2}$ & $2.36_{-1.0}^{+0.47}$ & 0.0359 & 0.3062 \\
ESO 187-0510 & 2 & $7.68_{-1.2}^{+0.62}$ & $3.39_{-0.64}^{+0.52}$ & 0.116 & 0.266 \\
ESO 302-0120 & 2 & $17.1_{-2.8}^{+2.2}$ & $3.38_{-0.83}^{+0.60}$ & 1.51 & 0.383 \\
ESO 305-0090 & 1 & $7.20_{-1.9}^{+0.53}$ & $2.05_{-0.52}^{+0.37}$ & 0.393 & 0.202 \\
ESO 425-0180 & 1 & $23.3_{-7.5}^{+2.2}$ & $1.37_{-0.41}^{+0.21}$ & 7.97 & 1.11 \\
ESO 488-0490 & 3 & $20.1_{-1.9}^{+1.2}$ & $5.99_{-0.88}^{+0.71}$ & 1.14 & 0.866 \\
UGC 4115 & 1 & $2.52_{-1.1}^{+0.35}$ & $14.1_{-3.0}^{+1.6}$ & 0.0402 & 0.086 \\
UGC 11557 & 1 & $16.4_{-7.2}^{+1.5}$ & $1.38_{-0.29}^{+0.16}$ & 1.04 & 0.400 \\
UGC 11583 & 4 & $8.32_{-1.8}^{+0.94}$ & $9.1_{-2.6}^{+1.4}$ & 0.046 & 0.176 \\
UGC 11819 & 2 & $22.6_{-0.84}^{+0.75}$ & $5.67 \pm 0.30$ & 5.21 & 0.987 \\
\hline
\end{tabular}
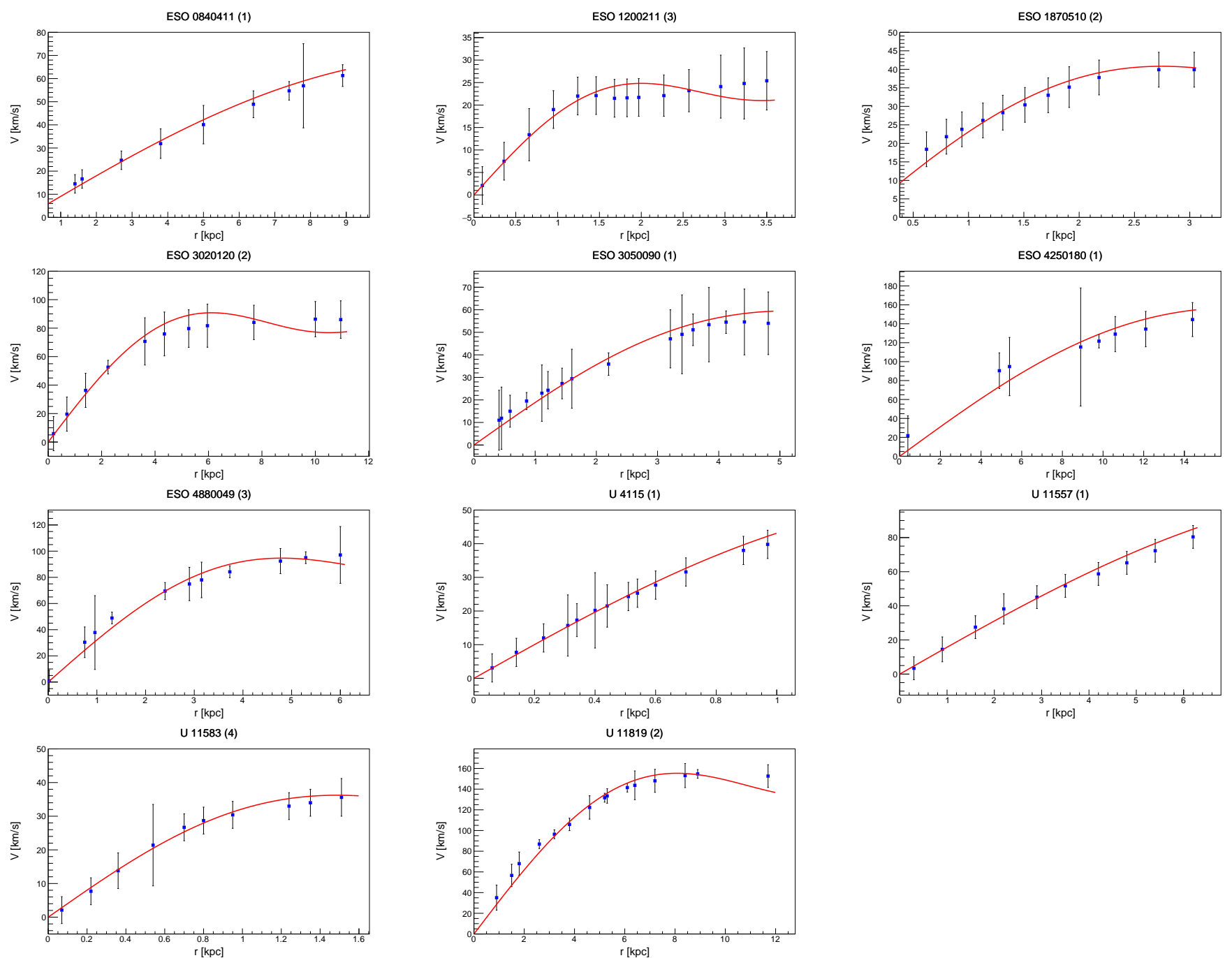

Figure 4. Best-fit profiles for the high-resolution LSB galaxies reported in Table 6, in the mSFDM model with one excited state $j$. 
Table 7. Multistate SFDM with two excited states in high-resolution LSB galaxies. In this Table we show the fitting parameters $R$, $\rho_{0}^{i}$ and $\rho_{0}^{j}$ for two excited states $i, j, \pm 1 \sigma$ errors from the MCMC method used, in the mSFDM model (10) for 7 high-resolution LSB galaxies in de Blok et al. (2001). We report the resulting DM masses, $M_{i}$ and $M_{j}$, for each state, the mass ratio $\eta=M_{j} / M_{1}$ (where the ground state $j=1$ appears in the fit) and $\chi_{\text {red }}^{2}$ errors from the fitting method.

\begin{tabular}{|c|c|c|c|c|c|c|c|c|}
\hline Galaxy & $i, j$ & $\begin{array}{c}R \\
(\mathrm{kpc})\end{array}$ & $\begin{array}{c}\rho_{0}^{i} \\
\left(10^{-2} M_{\odot} / \mathrm{pc}^{3}\right)\end{array}$ & $\begin{array}{c}\rho_{0}^{j} \\
\left(10^{-2} M_{\odot} / \mathrm{pc}^{3}\right)\end{array}$ & $\begin{array}{c}M_{i} \\
\left(10^{10} M_{\odot}\right)\end{array}$ & $\begin{array}{c}M_{j} \\
\left(10^{10} M_{\odot}\right)\end{array}$ & $\eta=M_{j} / M_{1}$ & $\chi_{\text {red }}^{2}$ \\
\hline ESO 014-0040 & 2,7 & $48.1_{-4.4}^{+2.6}$ & $2.93_{-0.89}^{+0.71}$ & $19.3_{-3.7}^{+3.1}$ & 26.9 & 16.0 & - & 0.206 \\
\hline ESO 206-0140 & 2,7 & $24.3_{-2.5}^{+1.4}$ & $\begin{array}{r}-0.89 \\
1.85_{-0.53}^{+0.37}\end{array}$ & $16.6_{-3.0}^{+2.1}$ & 2.11 & 1.43 & - & 0.275 \\
\hline F730-V1 & 1,4 & $15.6_{-2.6}^{+1.5}$ & $1.89_{-0.69}^{+0.49}$ & $17.4_{-3.3}^{+2.3}$ & 4.20 & 1.97 & 0.469 & 1.28 \\
\hline UGC 11454 & 1,3 & $14.56_{-1.1}^{+0.72}$ & $2.40 \pm 0.37$ & $10.4 \pm 1.1$ & 4.54 & 1.81 & 0.399 & 0.322 \\
\hline UGC 11616 & 2,5 & $25.8^{+2.1} .9$ & $2.07^{+0.42}$ & $10.62^{+0.95}$ & 2.56 & 1.84 & - & 0.597 \\
\hline UGC 11648 & 1,7 & $21.1_{-2.4}^{+1.5}$ & $\begin{array}{r}-0.72 \\
1.07_{-0.19}^{+0.12}\end{array}$ & $20.5_{-2.9}^{+1.2}$ & 4.48 & 1.45 & 0.324 & 0.698 \\
\hline UGC 11748 & 6,9 & $41.2_{-1.6}^{+1.4}$ & $28.0 \pm 5.8$ & $31.9 \pm 7.8$ & 17.3 & 9.11 & - & 1.68 \\
\hline
\end{tabular}

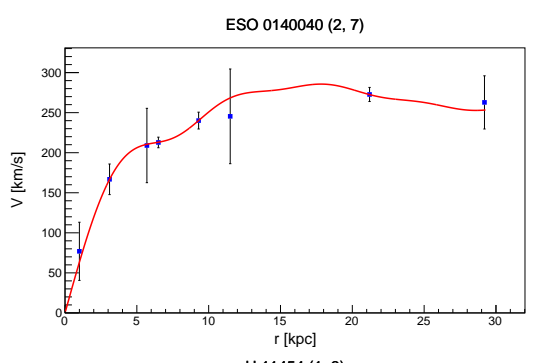

U $11454(1,3)$

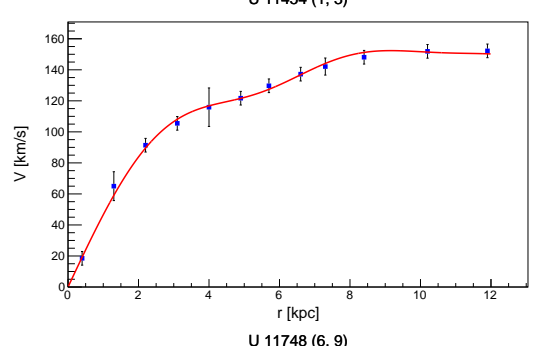

U $11748(6,9)$

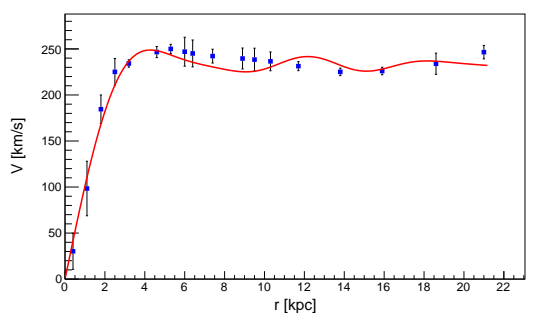

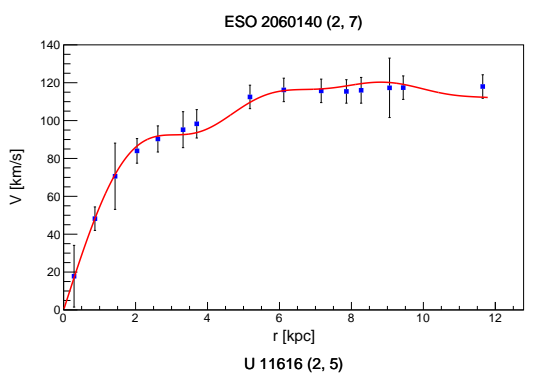
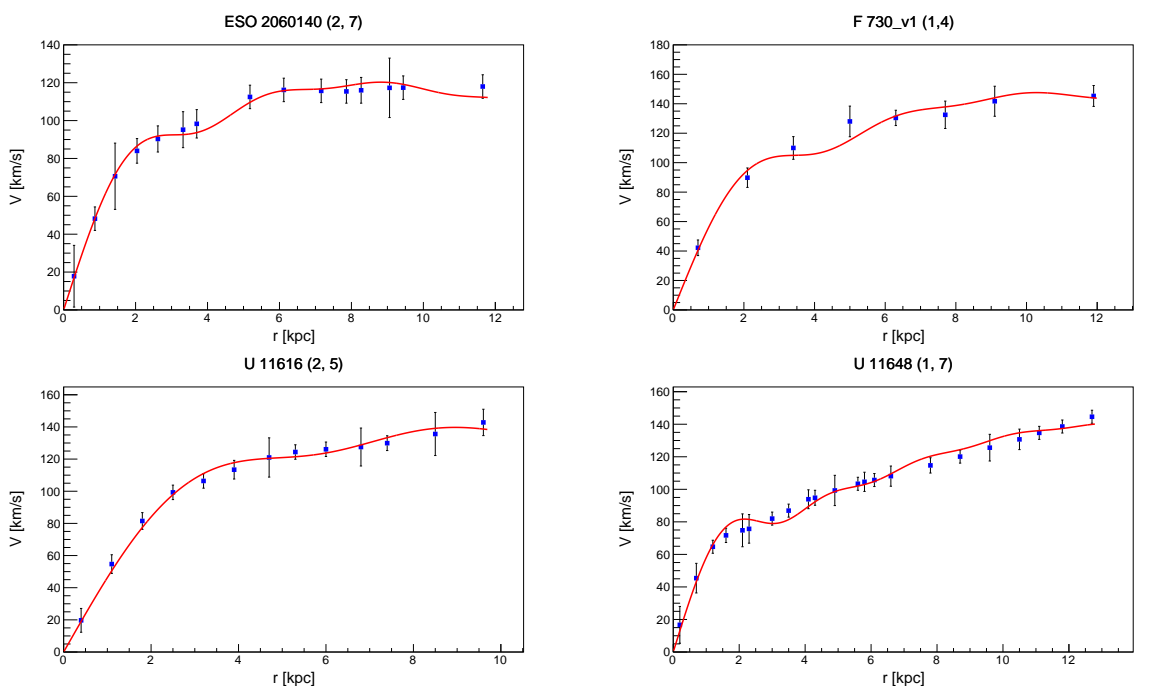

Figure 5. Best-fit profiles for the high-resolution LSB galaxies reported in Table 7 in the mSFDM model with two excited states $(i, j)$.

ies with $j=1$ and 2 with excited states only. In the case of the galaxies with a ground state, the values of the mass ratios are $\eta \lesssim 1.3$, consistent with the stability condition mentioned before, found through numerical simulations of multistate configurations (Ureña-López \& Bernal 2010).

Fig. 6 shows the resulting profiles with these fitting parameters, without and taking into account the baryonic information. For NGC 7814, the bulge-dominated galaxy, once the photometric information is included, the model reproduces very well the observations. For NGC 6503, the disk-dominated galaxy, the best fit fails at reproducing some regions, even taking into account the baryonic data; for this galaxy we used three excited states. For NGC 3741, the gasdominated galaxy, and for NGC 1560, both fits are very good. For NGC 1003 and 6946 the inner regions are reproduced once the baryonic information is included. For NGC 1003, the mSFDM model reproduces very well the 'wiggles' or oscillations, which are not reproduced by the baryonic components (see the previous results in Robles \& Matos 2013b), neither the soliton+NFW profile (see Subsection 4.1.3).

In Table 9, we present the best-fit three states configuration for NGC 6503, for the data without and with photometry, and the resulting rotation curves are in Fig. 7. For the other galaxies, the fittings might be improved by introducing a third excited state, but is not necessary to introduce more degrees of freedom since two excited states are well enough. It is worth noting that in these cases the other intermedi- 
Table 8. Multistate SFDM with two excited states in NGC galaxies with photometric data. The top panel shows the DM-only fits and the bottom panel the DM+baryons analysis, both for the same sample galaxies with high-resolution photometric information. Both panels show the fitting parameters $R, \rho_{0}^{i}$ and $\rho_{0}^{j}$ for two excited states $i, j, \pm 1 \sigma$ errors from the MCMC method used, in the multistate SFDM model (10). We also report the resulting DM masses, $M_{i}$ and $M_{j}$, for each state, the mass ratio $\eta=M_{j} / M_{1}$ and $\chi_{\text {red }}^{2}$ errors from the fitting method.

\begin{tabular}{|c|c|c|c|c|c|c|c|c|}
\hline \multicolumn{9}{|c|}{ DM-only fits } \\
\hline Galaxy & $i, j$ & $\begin{array}{c}R \\
(\mathrm{kpc})\end{array}$ & $\begin{array}{c}\rho_{0}^{i} \\
\left(10^{-2} M_{\odot} / \mathrm{pc}^{3}\right)\end{array}$ & 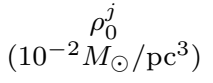 & $\begin{array}{c}M_{i} \\
\left(10^{10} M_{\odot}\right)\end{array}$ & $\begin{array}{c}M_{j} \\
\left(10^{10} M_{\odot}\right)\end{array}$ & $\eta=M_{j} / M_{1}$ & $\chi_{\text {red }}^{2}$ \\
\hline NGC 7814 & 9 & $11.25_{-0.12}^{+0.10}$ & - & $1122_{-22}^{+24}$ & - & 22.0 & - & 31.7 \\
\hline NGC 6503 & 3,9 & $19.30_{-0.43}^{+0.54}$ & $3.79_{-0.65}^{+0.55}$ & $73.5_{-6.5}^{+5.2}$ & 2.429 & 5.08 & - & 2.35 \\
\hline NGC 3741 & 1,7 & $9.04_{-0.76}^{+0.49}$ & $0.774 \pm 0.096$ & $10.7_{-1.3}^{+1.5}$ & 0.334 & 0.0783 & 0.234 & 0.33 \\
\hline NGC 1003 & 1,4 & $43.41 \pm 0.61$ & $0.0717 \pm 0.0045$ & $2.470 \pm 0.078$ & 3.279 & 6.01 & 1.83 & 1.46 \\
\hline NGC 1560 & 1,3 & $13.88 \pm 0.46$ & $0.574_{-0.042}^{+0.035}$ & $3.10 \pm 0.13$ & 0.814 & 0.391 & 0.48 & 0.61 \\
\hline NGC 6946 & 2,3 & $42.28 \pm 0.90$ & $0.89 \pm 0.12$ & $2.96 \pm 0.12$ & 6.901 & 10.6 & - & 1.40 \\
\hline \multicolumn{9}{|c|}{ DM+baryons fits } \\
\hline NGC 7814 & 4,9 & $103.9_{-7.0}^{+5.8}$ & $0.88_{-0.15}^{+0.11}$ & $3.85_{-0.48}^{+0.36}$ & 8.818 & 6.902 & - & 0.388 \\
\hline NGC 6503 & 2,7 & $44.26 \pm 0.88$ & $0.318 \pm 0.024$ & $5.26 \pm 0.20$ & 2.194 & 3.270 & - & 4.05 \\
\hline NGC 3741 & 1,6 & $8.99_{-0.75}^{+0.52}$ & $0.730_{-0.10}^{+0.085}$ & $5.75_{-0.89}^{+0.75}$ & 0.306 & 0.059 & 0.19 & 0.209 \\
\hline NGC 1003 & 1,4 & $44.84_{-1.2}^{+0.88}$ & $0.0912 \pm 0.0050$ & $1.488_{-0.068}^{+0.077}$ & 4.442 & 3.933 & 0.885 & 0.527 \\
\hline NGC 1560 & 1,3 & $14.77 \pm 0.61$ & $0.417_{-0.037}^{+0.030}$ & $2.23 \pm 0.12$ & 0.661 & 0.338 & 0.511 & 0.488 \\
\hline NGC 6946 & 1,3 & $38.19 \pm 0.99$ & $0.199 \pm 0.015$ & $2.035 \pm 0.079$ & 6.559 & 5.775 & 0.88 & 0.374 \\
\hline
\end{tabular}

ate states (including the ground state) might be present in the mSFDM halo, but their contribution to the total density profile is negligible with respect to the dominant ones.

Finally, Fig. 8 illustrates the posterior distributions and $1 \sigma$ and $2 \sigma$ contours obtained from the MCMC method for NGC 6503, with two and three excited states. The parameter $R$ is the scatter plot.

\section{DISCUSSION AND CONCLUSIONS}

From the cosmological FDM simulations it is found that the galaxy halos are formed by prominent dense cores, that approximate well the soliton-like solutions of the SchrödingerPoisson equation. However, the soliton profile alone is unable to reproduce the rotation curves, in particular of large galaxies. It is in this case where is necessary to introduce a more general profile than the soliton one in order to reproduce the observations at the outer radii of large systems. An ad hoc approach smoothly matches the soliton solution with a NFW profile (soliton+NFW) (Schive et al. 014a,b). On the other side, a theoretically motivated alternative that has been proposed in the literature is to include excited states in the SFDM halo (multistate SFDM) coming from thermal excitations in the SF potential (Robles \& Matos 2013b), that presents a soliton core-like behavior at the innermost radii and 'wiggles' or oscillations at the outer radii in the density profile and rotation curves (see Fig. 10). In this article we take both alternatives, and apply the soliton+NFW profile and the mSFDM model to reproduce the rotation curves of 18 LSB galaxies and 6 NGC galaxies with high-resolution photometric data.

In the first place, we apply the soliton $+\mathrm{NFW}$ profile with the continuity condition between both profiles at the transition radius $r_{\epsilon}$. For all the galaxies, from the individual fitting analysis leaving vary freely the four para- meters of the model (boson mass $m_{\psi}$, soliton core radius $r_{c}$, transition radius $r_{\epsilon}$ and characteristic NFW radius $r_{s}$ ), we found that the resulting boson mass is in the range $0.212 \leqslant m_{\psi} / 10^{-23} \mathrm{eV} \leqslant 27.0$, the core radius within $0.326 \leqslant r_{c} / \mathrm{kpc} \leqslant 8.96$, and the ratio between the transition and core radii in $0.46 \leqslant r_{\epsilon} / r_{c} \leqslant 1.94$ (see Tables 3 and 5 ). However, as the boson mass must be a constant of the FDM model, we performed a combined analysis of the 18 LSB galaxies for a single boson mass for all the galaxies, and obtained $m_{\psi}=0.554 \times 10^{-23} \mathrm{eV}$, with core radius in the range $1.57 \leqslant r_{c} / \mathrm{kpc} \leqslant 5.82$, and ratio between the transition and core radii in the interval $0.15 \leqslant r_{\epsilon} / r_{c} \leqslant 2.3$ (see Table 4). We point out that we are not reporting the confidence interval for the boson mass in this analysis since the combined computation time was too expensive. However, we believe that the confidence level is not too large based on the boson mass errors reported in Table 3 for the individual analysis, and also given the value of $\chi_{\text {red }}^{2}=1.208$ obtained in the combined analysis.

Remarkably, in both the individual and the combined analysis, in order to fit the rotation curves of the galaxies, we obtained different results from the expected $r_{\epsilon}>3 r_{c}$ in the FDM cosmological simulations (Schive et al. 014a). Furthermore, in the combined analysis we obtained a best-fit mass $m_{\psi}<10^{-23} \mathrm{eV}$, that is in conflict with the stringent cosmological constraints (Bozek et al. 2015; Sarkar et al. 2016), setting up a possible tension between the FDM model and the galaxy rotation curves. As it is known, in the SFDM there is a sharp break in the matter power spectrum leading to a natural suppression of substructure below a scale dependent on the boson mass, $k \sim m_{\psi}^{1 / 3}$. The cosmological implications for such light boson masses $\left(m_{\psi}<10^{-23} \mathrm{eV}\right)$ would imply less substructure than observed. For these galaxies, we expected that the range in core radius will decrease as the boson mass increases to have $m_{\psi}>10^{-23} \mathrm{eV}$, once the cosmological restriction was imposed; however, if we restrict 


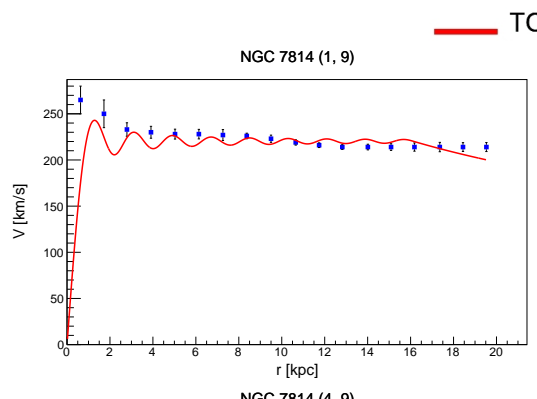

TOTAL RC $=\ldots \ldots$ STARS $\ldots$ BULGE $\ldots=\ldots$ GAS mSFDM
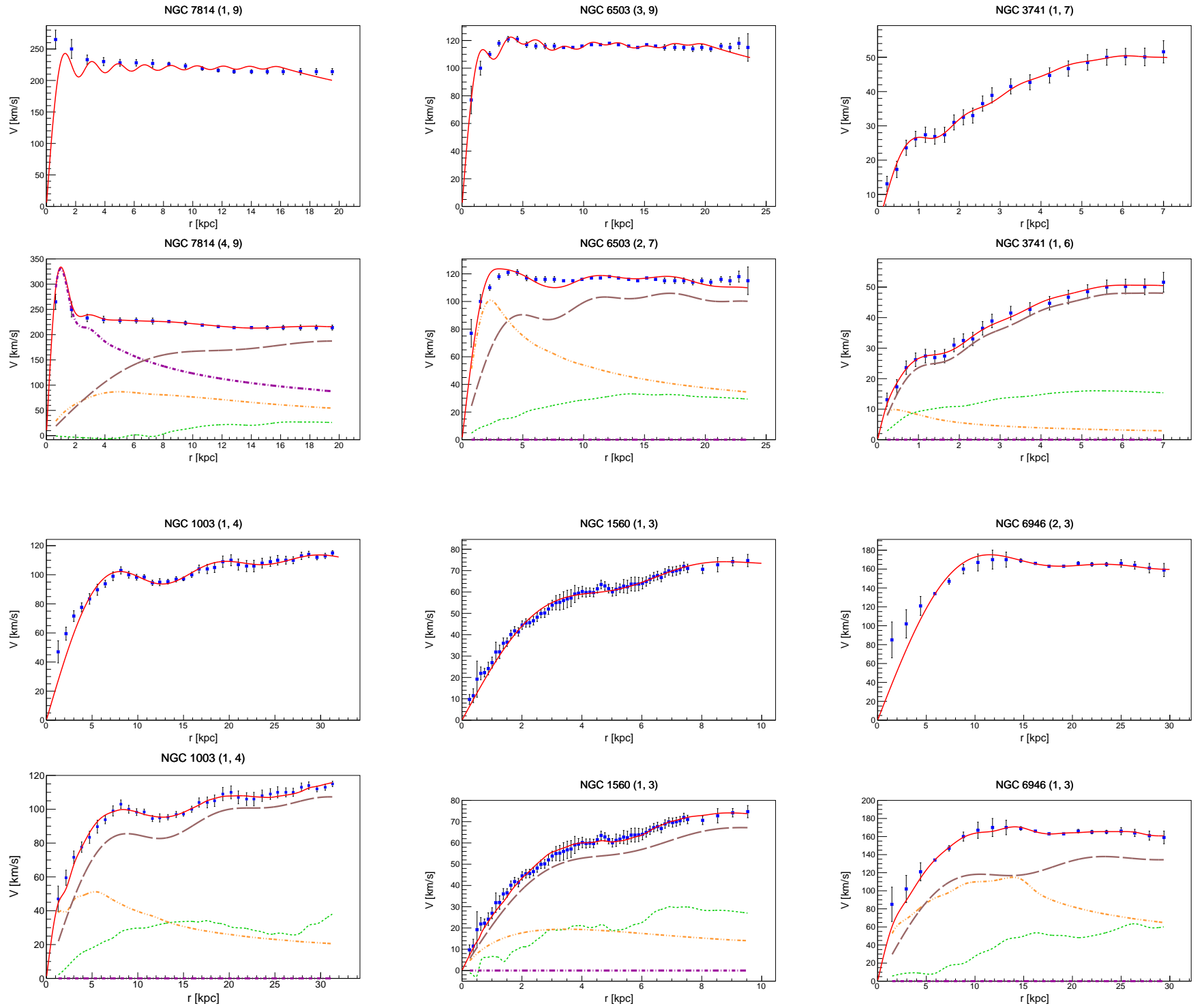

Figure 6. Best fits for the NGC galaxies with photometric data in the mSFDM model with two excited states $(i, j)$, for DM-only and $\mathrm{DM}+$ baryons contribution. The corresponding fitting parameters are shown in Table 8.

Table 9. Multistate SFDM with three excited states in NGC 6503. The top panel shows the DM-only fit and the bottom panel the $\mathrm{DM}+$ baryons analysis. Both panels show the fitting parameters $R, \rho_{0}^{i}, \rho_{0}^{j}$ and $\rho_{0}^{k}$ for three excited states $i, j, k, \pm 1 \sigma$ errors from the MCMC method. We also report the resulting DM masses, $M_{i}, M_{j}$ and $M_{k}$, for each state and $\chi_{\text {red }}^{2}$ errors from the fitting method.

\begin{tabular}{|c|c|c|c|c|c|c|c|c|c|}
\hline \multicolumn{10}{|c|}{ DM-only fit } \\
\hline Galaxy & $i, j, k$ & $\begin{array}{c}R \\
(\mathrm{kpc})\end{array}$ & $\begin{array}{c}\rho_{0}^{i} \\
\left(10^{-2} M_{\odot} / \mathrm{pc}^{3}\right)\end{array}$ & $\begin{array}{c}\rho_{0}^{j} \\
\left(10^{-2} M_{\odot} / \mathrm{pc}^{3}\right)\end{array}$ & $\begin{array}{c}\rho_{0}^{k} \\
\left(10^{-2} M_{\odot} / \mathrm{pc}^{3}\right)\end{array}$ & $\begin{array}{c}M_{i} \\
\left(10^{10} M_{\odot}\right)\end{array}$ & $\begin{array}{c}M_{j} \\
\left(10^{10} M_{\odot}\right)\end{array}$ & $\begin{array}{c}M_{k} \\
\left(10^{10} M_{\odot}\right)\end{array}$ & $\chi_{\mathrm{red}}^{2}$ \\
\hline NGC 6503 & $4,5,9$ & $24.2 \pm 0.48$ & $4.37 \pm 0.63$ & $5.56 \pm 0.87$ & $27.98 \pm 3.3$ & 2.456 & 2.000 & 3.082 & 1.27 \\
\hline \multicolumn{10}{|c|}{ DM+baryons fit } \\
\hline NGC 6503 & $2,4,7$ & $50.0_{-2.5}^{+2.0}$ & $0.197 \pm 0.032$ & $0.671_{-0.082}^{+0.066}$ & $2.89_{-0.29}^{+0.24}$ & 1.957 & 1.659 & 2.103 & 1.54 \\
\hline
\end{tabular}



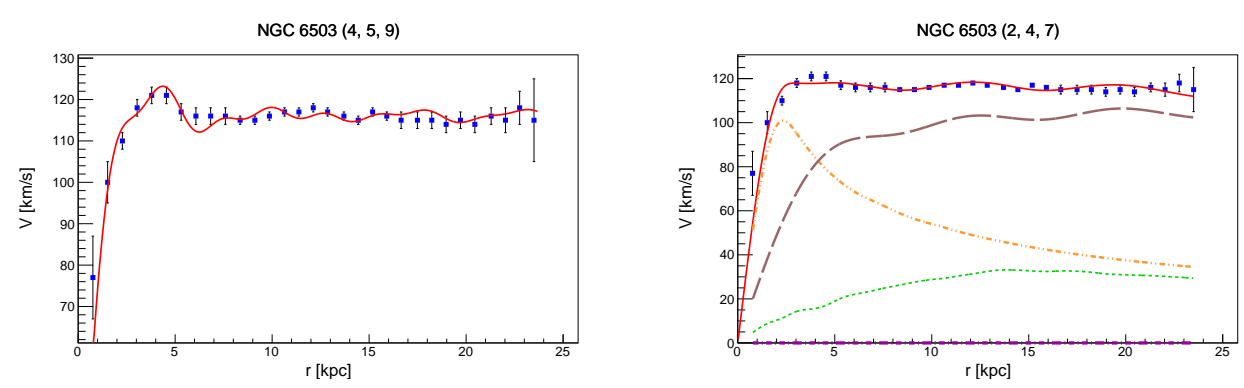

Figure 7. The same as Fig. 6 for NGC 6503 with three excited states $(i, j, k)$. The fitting parameters are reported in Table 9.
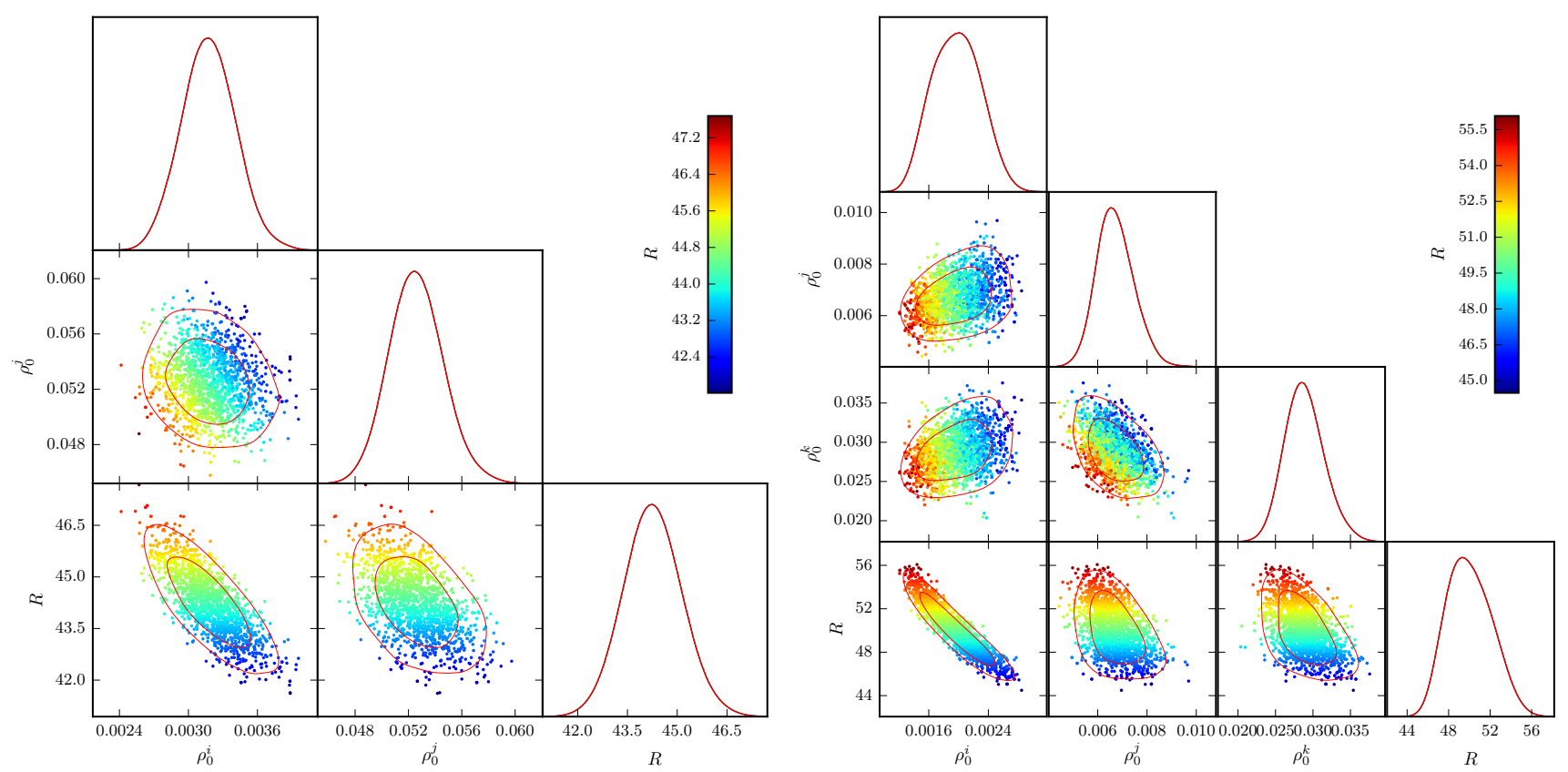

Figure 8. Posterior distributions for the parameters $\rho_{0}^{i}\left(M_{\odot} / \mathrm{pc}^{3}\right), \rho_{0}^{j}\left(M_{\odot} / \mathrm{pc}^{3}\right)$ and $R(\mathrm{kpc})$, with $R$ as the scatter plot, for the two (left panel) and three (right panel) states configurations for NGC 6503.

the fitting methods to obtain $m_{\psi} \geqslant 10^{-23} \mathrm{eV}$ it is not possible to fit all the rotation curves together, so we did not include those results in the paper.

In Fig. 9, we show the resulting distribution of core radii $r_{c}$ vs. boson masses $m_{\psi}$ for all the galaxies in the individual analysis, $\pm 1 \sigma$ errors from the MCMC method. The solid line in the figure, at $m_{\psi}=0.554 \times 10^{-23} \mathrm{eV}$, shows the result for the boson mass from the combined analysis with the LSB galaxies, and the dashed line shows the minimum ultra-light boson mass required from the cosmological constraints. Notice the correlation between both parameters: for large galaxies the core radius is larger and the resulting boson mass is smaller, and vice versa, for smaller galaxies the core radius is smaller and the boson mass larger. Such correlation has been discussed very recently in Ureña-López et al. (2017), including dSph galaxies' results (whose $R_{\max } \sim 0.5-2 \mathrm{kpc}$ ), for which the estimated boson mass from the Jeans analysis of the classical dSphs is $m_{\psi}=1.79 \times 10^{-22} \mathrm{eV}$ (Chen et al. 2017), and from a detailed analysis using kinematic mock data of Fornax and Sculptor is $m_{\psi}=2.4 \times 10^{-22} \mathrm{eV}$ (González-Morales et al.
2016), two orders of magnitude larger than the value obtained from the combined analysis of the LSB galaxies in this work. In our case, the galaxies span a wide range in sizes $\left(R_{\max } \sim 1-30 \mathrm{kpc}\right)$, thus there is large scattering for both $r_{c}$ and $m_{\psi}$, and it is expected that for large galaxies the resulting boson mass will be smaller than $10^{-23} \mathrm{eV}$.

As noted in Ureña-López et al. (2017), this could happen because the parameters of the FDM model, including the boson mass and soliton core radius, are allowed to vary freely, thus the value of $m_{\psi}$ will depend on the properties of the sample and the fitting methods will not necessarily imply the FDM model is wrong in these systems. This kind of fitting methods could not provide a reliable determination of the boson mass $m_{\psi}$, and this could be a possible explanation of the variety of results coming from different astronomical observations (see Subsection 2.1). Based on the mass discrepancy-acceleration relation (McGaugh et al. 2016; Lelli et al. 2017), Ureña-López et al. (2017) proposed there is a universal central surface density for any DM profile, leading to a correlation between its central density and scale radius (see also Garcia-Aspeitia et al. 2017). In the SFDM model, 


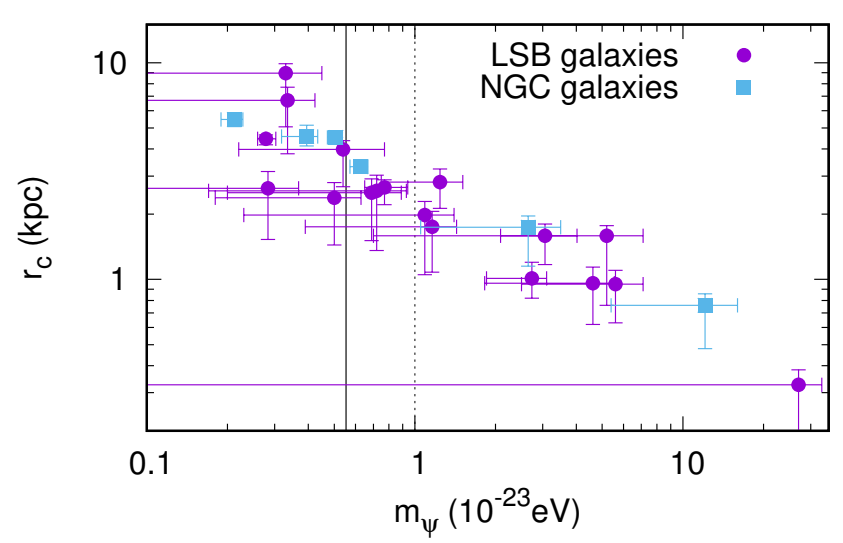

Figure 9. Distribution of resulting core radii $r_{c}$ vs. boson masses $m_{\psi}$ from the soliton+NFW density profile in the FDM model, for the individual analysis of the 18 LSB and 6 NGC (including baryonic information) galaxies, $\pm 1 \sigma$ errors from the MCMC method. The vertical dashed line at $m_{\psi}=10^{-23} \mathrm{eV}$ shows the cosmological constraint (Bozek et al. 2015; Sarkar et al. 2016) for the minimum mass required for the ultra-light boson. The vertical solid line at $m_{\psi}=0.554 \times 10^{-23} \mathrm{eV}$ shows the result for the boson mass from the combined analysis of the 18 LSB galaxies.

this implies that all the galaxy halos should have a universal soliton structure at the galaxy centers, with a constant boson mass $m_{\psi}=1.2 \times 10^{-21} \mathrm{eV}$, that is in agreement with the severe cosmological constraints $\left(m_{\psi}>10^{-23} \mathrm{eV}\right)$. This universal soliton should have a core radius $r_{c} \approx 0.3 \mathrm{kpc}$ and total mass $M_{s}=1.8 \times 10^{7} M_{\odot}$. Those authors analyzed the complete soliton+NFW profile and found the results are in agreement with the universal soliton profile solution with a constant boson mass $m_{\psi} \sim 10^{-21} \mathrm{eV}$, with surface densities close to the soliton-only results and $r_{c} \sim 0.3 \mathrm{kpc}$. These results suggest the rotation curves of a diverse sample of galaxies might be fitted with this large mass, leaving open the possibility to alleviate the tension between the soliton $+\mathrm{NFW}$ profile and the large galaxies observations. However, from our results in this article, we found instead $m_{\psi}<10^{-23} \mathrm{eV}$. A more detailed study of large galaxies including the universal soliton hypothesis, is needed.

On the other hand, we apply the exact mSFDM model, that includes self-interactions and multistate solutions coming from thermal excitations in the SF potential, as a realistic SFDM halo. In this work, we find that the model is consistent with the rotation curves for both LSB and NGC galaxies with photometric information. For one excitation state in equation (10) (with the excited state $i$, the radius of the halo $R$ and the central density $\rho_{0}^{i}$ as free parameters), the $\chi_{\text {red }}^{2}$ errors are smaller than those of the soliton+NFW profile for 6 of the 11 LSB galaxies fitted, almost the same for 4 and larger for 1 . In this case, it was possible to fit the galaxies with the ground state only $(j=1)$ for almost the half of the sample. For combinations of two excited states, the $\chi_{\text {red }}^{2}$ errors are smaller for the mSFDM profile compared to the soliton+NFW results for the other 7 LSB galaxies, and for 5 of the 6 NGCs with photometric data. For these fits, the ground state is present in more than a half of the samples. Finally, in order to improve the fit for NGC 6503, we used three excited states. Notice the freedom in choosing the excitation states $i, j$ in order to fit the observations within the mSFDM model; in principle, the excitation state $j$ can take any value $j=1,2,3, \ldots$, however, we preferred lower states since, in this scenario, it is expected that the mSFDM halos evolve from BEC configurations with all bosons in the ground state, thus it would be easier to reach the lower energy levels first.

For the resulting two-states-configurations in the multistate SFDM model, we assume the stability threshold by Ureña-López \& Bernal (2010), where for multistate systems where the mass ratios $\eta=M_{j} / M_{1} \lesssim 1.3$, with respect to the ground state $M_{1}=M_{\mathrm{mSFDM}}(j=1)$, the excited state $j$ does not decay to the ground state, i.e. is a stable multistate configuration. In our fits, we obtained 3 of 7 LSB galaxies and 4 of 6 NGC galaxies with baryonic data with a dominant ground state $j=1$; for these multistate galaxies we argue that they might be stable systems since the mass ratios satisfy such numerical restriction. However, further numerical simulations including the baryonic components are needed to investigate the stability of the multistate configurations and the influence of the baryonic matter in the mSFDM halo. Moreover, for the galaxies with excited states only $(j>1)$, the final distribution in different excitation levels might be the final product of galaxy formation processes and interactions with the baryonic matter, and numerical simulations are required in order to investigate the stability of such configurations.

Finally, Fig. 10 shows the resulting soliton + NFW and mSFDM density profiles for three sample LSB galaxies. There is an evident overlap between both profiles, showing the core-like behavior at the innermost radii, the oscillations at intermediate radii (inherent to the mSFDM model) around the $r^{-3} \mathrm{NFW}$ decline, but a steeper slope of the mSFDM with respect to the NFW decline at the outermost radii, defining more compact $\mathrm{mSFDM}$ halos due to the election of the radius $R$. Such overlap for the outer regions was already discussed in Bernal et al. (2017), from the results of fitting the mSFDM model and the NFW profile to the X-ray observations of clusters of galaxies, noticing the core-like behavior that the mSFDM profile should share with the soliton core density. Such overlap of the NFW and SFDM profiles was also seen in recent simulations of mergers of FDM halos in the ground state (Schive et al. 014a; Schwabe et al. 2016; Mocz et al. 2017). Furthermore, we notice the similar behavior of the oscillations of the mSFDM around the NFW profile and the numerical simulations of solitonic core mergers in Schwabe et al. (2016). The final merger configurations display new 'excited' solitonic cores. One possible interpretation of such excited cores is that those are the mSFDM solutions at the outer radii after the merger simulations, as a realistic scenario where the SF does not remain in the soliton DM cores exclusively, after galaxy and galaxy clusters formation processes, showing excitation states corresponding to multistate solutions.

To conclude, we obtained the ultra-light boson mass required to fit the rotation curves of LSB and NGC galaxies in the context of the FDM model. The combined analysis of the LSB galaxies gives the value $m_{\psi}=0.554 \times 10^{-23} \mathrm{eV}$, setting up a tension of the model with the cosmological constraints for the minimum boson mass required to reproduce the observed substructure. As an interesting alternative, the ana- 


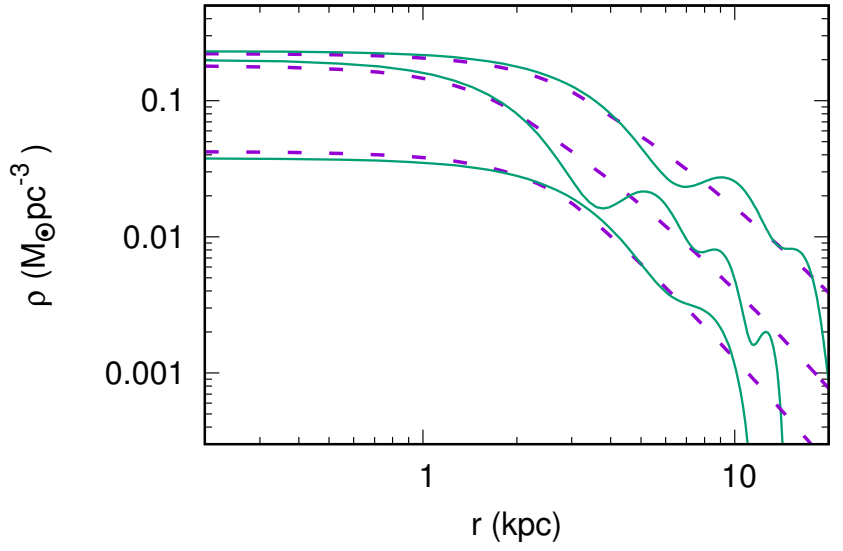

Figure 10. Resulting density profiles for three sample LSB galaxies. The dashed lines show the soliton+NFW profiles and the solid lines the corresponding mSFDM fits. The overlap between both profiles is evident for the galaxies, showing the core-like behavior at the innermost radii, the 'wiggles' or oscillations around the NFW-region, inherent to the multistate profile, and the steeper slope at the outermost radii of the mSFDM model compared to the NFW profile. Every galaxy shows different oscillations depending on its particular multistate configuration.

lytic mSFDM solution is successful in reproducing the observations of dwarf and larger galaxies and galaxy clusters, in particular the wiggles or oscillations observed in some systems. Even when, at this point, with the mSFDM model it is not possible to constrain the boson properties, the ultra-light mass $m_{\psi}$ and the self-interaction $\lambda$, we show that this model is a theoretically motivated framework for realistic SFDM halos, and as an analytic solution can be used, at least, as a useful fitting-function in several astrophysical systems and along with, or as a substitute of the empirical soliton $+\mathrm{NFW}$ profile.

Unfortunately, in the analysis we performed here, we are in fact neglecting processes in the galaxies that are somehow important, like supernovae explosions, star formation, stellar winds, etc. This processes give some uncertainties to the observations compared with the SFDM profiles, and it is one of the main reasons we are not able to fit the boson mass with better accuracy with these kind of fitting methods.

\section{ACKNOWLEDGEMENTS}

We gratefully acknowledge Stacy McGaugh for providing us the observational data used in this article and for helpful comments and discussions about his work in previous publications. We thank Luis Arturo Ureña-López and Victor Robles who provided insights and suggestions for the final results in this article, and the referee who reviewed carefully the previous version and help us to strengthen the results in the final version. This work was partially supported by CONACyT México under grants CB-2011 No. 166212, CB-2014-01 No. 240512, Project No. 269652 and Fronteras Project 281; Xiuhcoatl and Abacus clusters at Cinvestav, IPN; I0101/131/07 C-234/07 of the Instituto Avanzado de Cosmología (IAC) collaboration (http://www.iac.edu.mx).

\section{APPENDIX A: SOLITON+NFW MASS PROFILE}

For the soliton $+\mathrm{NFW}$ profile (4) the density is restricted to be continuous at the transition radius $r_{\epsilon}$ (Schive et al. 014a; Marsh \& Pop 2015):

$\rho_{\mathrm{sol}}\left(r_{\epsilon}\right)=\rho_{\mathrm{NFW}}\left(r_{\epsilon}\right)$.

With this condition, we have four free parameters, $\rho_{c}, r_{c}, r_{\epsilon}$ from the soliton density (5) and $r_{s}$ from the NFW profile (6). The NFW density parameter $\rho_{s}$ can be written as

$\rho_{s}\left(\rho_{c}, r_{c}, r_{\epsilon}, r_{s}\right)=\rho_{c} \frac{\left(r_{\epsilon} / r_{s}\right)\left(1+r_{\epsilon} / r_{s}\right)^{2}}{\left[1+0.091\left(r_{\epsilon} / r_{c}\right)^{2}\right]^{8}}$,

where $\rho_{c}:=1.9\left(m_{\psi} / 10^{-23} \mathrm{eV}\right)^{-2}\left(r_{c} / \mathrm{kpc}\right)^{-4}$.

The total soliton + NFW mass is given by

$M_{\mathrm{FDM}}(r)=\left\{\begin{array}{ll}M_{\mathrm{sol}}(r), & \text { if } r \leqslant r_{\epsilon} \\ M_{\mathrm{sol}}\left(r_{\epsilon}\right)-M_{\mathrm{NFW}}\left(r_{\epsilon}\right)+M_{\mathrm{NFW}}(r), & \text { if } r>r_{\epsilon}\end{array} ;\right.$

where the core mass function is (Chen et al. 2017)

$$
\begin{aligned}
& M_{\mathrm{sol}}(r)=\frac{4.2 \times 10^{6} M_{\odot}}{\left(m_{\psi} / 10^{-23} \mathrm{eV}\right)^{2}\left(r_{c} / \mathrm{kpc}\right)} \frac{1}{\left(1+a^{2}\right)^{7}} \\
& {\left[3465 a^{13}+23100 a^{11}+65373 a^{9}+101376 a^{7}+92323 a^{5}\right.} \\
& \left.+48580 a^{3}-3465 a+3465\left(1+a^{2}\right)^{7} \arctan a\right], \quad(\mathrm{A} 4)
\end{aligned}
$$

with $a:=0.301\left(r / r_{c}\right)$, and the NFW mass profile is given by (Navarro et al. 1997)

$M_{\mathrm{NFW}}(r)=4 \pi \rho_{s} r_{s}^{3}\left[\ln \left(1+\frac{r}{r_{s}}\right)-\frac{r / r_{s}}{1+r / r_{s}}\right]$.

\section{APPENDIX B: SOLITON+NFW ANALYSIS WITH DIFFERENTIABILITY CONDITION}

Additionally to the performed analysis of the FDM model, we explored the soliton+NFW density profile (4) to be continuous (equation (A1)) and differentiable at the transition radius $r_{\epsilon}$ :

$\rho_{\mathrm{sol}}^{\prime}\left(r_{\epsilon}\right)=\rho_{\mathrm{NFW}}^{\prime}\left(r_{\epsilon}\right)$.

With these conditions, the two NFW parameters $r_{s}$ and $\rho_{s}$ can be written in terms of the other three free parameters, $\rho_{c}, r_{c}$ and $r_{\epsilon}$, as

$$
\begin{aligned}
r_{s}\left(r_{c}, r_{\epsilon}\right) & =\left(\frac{b-3}{1-b}\right) r_{\epsilon}, \\
\rho_{s}\left(\rho_{c}, r_{c}, r_{\epsilon}\right) & =\frac{1-b}{(b-3)^{3}} \frac{4 \rho_{c}}{\left[1+0.091\left(r_{\epsilon} / r_{c}\right)^{2}\right]^{8}},
\end{aligned}
$$

where $b:=1.456\left(r_{\epsilon} / r_{c}\right)^{2} /\left[1+0.091\left(r_{\epsilon} / r_{c}\right)^{2}\right]$.

In Table B1, we report the results of the individual analysis for the $18 \mathrm{LSB}$ galaxies with three free parameters, $\rho_{c}$, $r_{c}$ and $r_{\epsilon}$, and the resulting boson mass $m_{\psi}$, all the quantities $\pm 1 \sigma$ errors from the MCMC method, also the ratio $r_{\epsilon} / r_{c}$ and $\chi_{\text {red }}^{2}$ errors for each galaxy. Table B2 shows the results of the combined analysis for the same LSB galaxies.

From Table B1 it is noticeable that, in general, $r_{c}$ is of the order of the transition radius $r_{\epsilon}$. This means that the soliton contribution is overlapped with the NFW halo (as we ask for a smooth transition between both profiles), 
Table B1. In this Table we show the resulting parameters $\rho_{c}, r_{c}$ and $r_{\epsilon}$ for the FDM model with the soliton+NFW density profile (4) with the differentiability condition at the transition radius $r_{\epsilon}$, and the resulting boson mass $m_{\psi}$, all the quantities $\pm 1 \sigma$ errors from the MCMC method used. We also show the resulting $r_{200}$ from the NFW halo radius, concentration parameter $c$ and $\chi_{\text {red }}^{2}$ errors for the 18 high-resolution LSB galaxies in de Blok et al. (2001).

\begin{tabular}{|c|c|c|c|c|c|c|c|c|}
\hline Galaxy & $\begin{array}{c}\rho_{c} \\
\left(10^{-2} M_{\odot} / \mathrm{pc}^{3}\right)\end{array}$ & $\begin{array}{c}r_{c} \\
(\mathrm{kpc})\end{array}$ & $\begin{array}{c}r_{\epsilon} \\
(\mathrm{kpc})\end{array}$ & $r_{\epsilon} / r_{c}$ & $\begin{array}{c}m_{\psi} \\
\left(10^{-23} \mathrm{eV}\right)\end{array}$ & $\begin{array}{l}r_{200} \\
(\mathrm{kpc})\end{array}$ & $c$ & $\chi_{\mathrm{red}}^{2}$ \\
\hline ESO 014-0040 & $22.2_{-9.1}^{+4.4}$ & $3.12 \pm 0.76$ & $3.23 \pm 0.92$ & 1.04 & $0.352_{-0.16}^{+0.063}$ & 254 & 21.0 & 0.658 \\
\hline ESO 084-0411 & $0.588_{-0.15}^{+0.1}$ & $4.90_{-0.99}^{+1.5}$ & $4.0_{-16}^{+1.1}$ & 0.816 & $0.986_{-0.58}^{+0.011}$ & - & - & 0.092 \\
\hline ESO $120-0211$ & $2.66_{-0.91}^{+0.15}$ & $1.01_{-0.27}^{+0.17}$ & $1.44_{-0.78}^{+0.35}$ & 1.43 & $9.6_{-3.9}^{+1.68}$ & 21.5 & 44.5 & 0.074 \\
\hline ESO $187-0510$ & $4.66_{-1.4}^{+0.91}$ & $\begin{array}{l}-0.27 \\
1.20_{-0.34}^{+0.27}\end{array}$ & $1.46_{-0.84}^{+0.78}$ & 1.22 & $5.28_{-2.7}^{+0.9}$ & 37.7 & 21.1 & 0.101 \\
\hline ESO 206-0140 & $17.2_{-4.8}^{+3.4}$ & $1.65_{-0.36}^{+0.34}$ & $1.80_{-0.56}^{+0.84}$ & 1.09 & $1.34_{-0.49}^{+0.75}$ & 106 & 23.1 & 0.133 \\
\hline ESO $302-0120$ & $4.23_{-1.2}^{+0.8}$ & $\begin{array}{c}-0.36 \\
2.66 \pm 0.57\end{array}$ & $3.28_{-1.4}^{+0.86}$ & 1.23 & $1.09_{-0.48}^{+0.49}$ & 79.0 & 21.6 & 0.032 \\
\hline ESO 305-0090 & $2.65_{-0.99}^{+-1.2}$ & $2.26_{-0.89}^{+0.68}$ & $2.30_{-1.4}^{+1.4}$ & 1.02 & $2.589_{-1.9}^{+0.48}$ & - & - & 0.076 \\
\hline ESO $425-0180$ & $4.04_{-2.2}^{+0.99}$ & $3.7 \pm 1.1$ & $3.42_{-0.83}^{+1.4}$ & 0.924 & $0.653_{-0.35}^{+0.053}$ & 251 & 6.14 & 0.509 \\
\hline ESO $488-0490$ & $8.9_{-2.5}^{+1.4}$ & $1.98 \pm 0.45$ & $2.24_{-1.0}^{+0.83}$ & 1.13 & $1.37_{-0.62}^{+0.22}$ & 90.9 & 20.4 & 0.131 \\
\hline F730-V1 & $\begin{array}{r}-2.5 \\
18.1_{-5.0}^{+3.5}\end{array}$ & $1.84_{-0.46}^{+0.34}$ & $1.95_{-0.67}^{+0.039}$ & 1.06 & $1.08_{-0.44}^{+0.62}$ & 130 & 21.2 & 0.329 \\
\hline UGC 4115 & $\begin{array}{r}-5.0 \\
15.2_{-4.3}^{+2.0}\end{array}$ & $1.06_{-0.66}^{+0.146}$ & $1.037_{-0.99}^{-0.077}$ & 0.978 & $\begin{array}{l}6.5_{-5.8}^{+0.44} \\
+1.1\end{array}$ & 91.1 & 14.4 & 0.132 \\
\hline UGC 11454 & $\begin{array}{l}7^{-4.3} \\
15.7_{-4.0}^{+3.0}\end{array}$ & $\begin{array}{l}-0.66 \\
1.96_{-0.44}^{+0.39}\end{array}$ & $1.96_{-0.55}^{+0.49}$ & 1.00 & $\begin{array}{l}1.0_{-0.38}^{+0.81} \\
+0.21\end{array}$ & 157 & 16.0 & 0.364 \\
\hline UGC 11557 & $\begin{array}{l}-4.0 \\
1.91_{-0662}^{+0.18}\end{array}$ & $\begin{array}{l}1.0 .0 .44 \\
3.7_{-1.2}^{+1.4}\end{array}$ & $2.77_{-1 .}^{+0.95}$ & 0.749 & $1.152_{-0.83}^{-0.072}$ & - & - & 0.12 \\
\hline UGC 11583 & $9.5_{-2.6}^{+2.62}$ & $0.86_{-0.26}^{+-1.2}$ & $1.45_{-1.0}^{+0.1}$ & 1.69 & $7.2_{-3.0}^{+1.9}$ & - & - & 0.097 \\
\hline UGC 11616 & $\begin{array}{r}-2.6 \\
16.3_{-3.2}^{+1.9}\end{array}$ & $\begin{array}{c}-0.26 \\
1.93 \pm 0.28\end{array}$ & $2.10_{-0.49}^{+0.0}$ & 1.09 & $0.97_{-0.27}^{+0.0}$ & 122 & 22.4 & 0.189 \\
\hline UGC 11648 & $\begin{array}{r}-3.2 \\
226 \pm 25\end{array}$ & $0.0858_{-0.0093}^{+0.010}$ & $0.0737_{-0.0080}^{+0.098}$ & 0.859 & $128_{-30}^{+20}$ & 184 & 8.38 & 1.097 \\
\hline UGC 11748 & $97_{-23}^{+13}$ & $1.70_{-0.21}^{+0.0093}$ & $2.14 \pm 0.35$ & 1.26 & $\begin{array}{c}-30 \\
0.502_{-0.098}^{+0.056}\end{array}$ & 160 & 77.0 & 1.978 \\
\hline UGC 11819 & $7.10_{-0.96}^{+0.4}$ & $3.92_{-0.26}^{+0.21}$ & $4.9 \pm 1.1$ & 1.25 & $0.351_{-0.077}^{+0.098}$ & 139 & 28.0 & 0.28 \\
\hline
\end{tabular}

Table B2. Soliton + NFW density profile - Combined analysis in high-resolution LSB galaxies. In this Table we show the resulting fitting parameters $\rho_{c}, r_{c}, r_{\epsilon}$ and $r_{s}$ for the FDM model with the soliton+NFW density profile (4) with the differentiability condition, and the resulting boson mass $m_{\psi}=0.554 \times 10^{-23} \mathrm{eV}$. The combined analysis was performed minimizing the $\chi^{2}$ errors and we obtained $\chi_{\text {red }}^{2}=1.208$. We also show the resulting NFW density parameter $\rho_{s}$, the ratio $r_{\epsilon} / r_{c}$, the $r_{200}$ radius from the NFW halo, concentration parameter $c$ and total DM mass $M_{200}=M_{\mathrm{FDM}}\left(r_{200}\right)$, for the 18 high-resolution LSB galaxies in de Blok et al. (2001).

\begin{tabular}{|c|c|c|c|c|c|c|c|c|c|c|}
\hline Galaxy & $\begin{array}{c}\rho_{c} \\
\left(10^{-2} M_{\odot} / \mathrm{pc}^{3}\right)\end{array}$ & $\begin{array}{c}r_{c} \\
(\mathrm{kpc})\end{array}$ & $\begin{array}{c}r_{\epsilon} \\
(\mathrm{kpc})\end{array}$ & $r_{\epsilon} / r_{c}$ & $\begin{array}{c}m_{\psi} \\
\left(10^{-23} \mathrm{eV}\right)\end{array}$ & $\begin{array}{c}r_{s} \\
(\mathrm{kpc})\end{array}$ & $\begin{array}{c}\rho_{s} \\
\left(10^{-2} M_{\odot} / \mathrm{pc}^{3}\right)\end{array}$ & $\begin{array}{l}r_{200} \\
(\mathrm{kpc})\end{array}$ & $c$ & $\begin{array}{c}M_{200} \\
\left(10^{12} M_{\odot}\right)\end{array}$ \\
\hline ESO 014-0040 & 2.61 & 0.855 & 0.767 & 0.897 & 2.024 & 16.0 & 2.61 & 289 & 18.1 & 2.66 \\
\hline ESO 084-0411 & 0.0020 & 2.593 & 2.227 & 0.859 & 2.024 & 696 & 0.0020 & 412 & 0.592 & 7.76 \\
\hline ESO 120-0211 & 1.99 & 2.654 & 3.440 & 1.296 & 2.024 & 2.69 & 1.99 & 43.8 & 16.3 & 0.009 \\
\hline ESO 187-0510 & 192 & 2.013 & 3.018 & 1.499 & 2.024 & 0.499 & 192 & 45.7 & 91.7 & 0.009 \\
\hline ESO 206-0140 & 3.15 & 1.183 & 1.173 & 0.992 & 2.024 & 6.27 & 3.15 & 122 & 19.5 & 0.201 \\
\hline ESO 302-0120 & 0.549 & 1.654 & 1.584 & 0.958 & 2.024 & 12.0 & 0.549 & 117 & 9.70 & 0.176 \\
\hline ESO 305-0090 & 0.110 & 2.032 & 1.848 & 0.909 & 2.024 & 29.0 & 0.110 & 142 & 4.90 & 0.317 \\
\hline ESO 425-0180 & 0.0945 & 1.537 & 1.341 & 0.873 & 2.024 & 71.6 & 0.0945 & 328 & 4.59 & 3.92 \\
\hline ESO 488-0490 & 0.577 & 1.429 & 1.318 & 0.922 & 2.024 & 16.3 & 0.577 & 162 & 9.90 & 0.467 \\
\hline F730-V1 & 1.85 & 1.143 & 1.073 & 0.939 & 2.024 & 10.4 & 1.85 & 164 & 15.8 & 0.484 \\
\hline UGC 4115 & 7.17 & 1.387 & 1.601 & 1.155 & 2.024 & 2.78 & 7.17 & 74.5 & 26.8 & 0.045 \\
\hline UGC 11454 & 1.21 & 1.161 & 1.065 & 0.917 & 2.024 & 14.4 & 1.21 & 192 & 13.4 & 0.780 \\
\hline UGC 11557 & 0.0049 & 1.940 & 1.665 & 0.858 & 2.024 & 662 & 0.0049 & 693 & 1.05 & 36.8 \\
\hline UGC 11583 & 3.59 & 1.692 & 1.974 & 1.167 & 2.024 & 3.20 & 3.59 & 65.6 & 20.5 & 0.031 \\
\hline UGC 11616 & 1.85 & 1.152 & 1.084 & 0.941 & 2.024 & 10.2 & 1.85 & 161 & 15.8 & 0.459 \\
\hline UGC 11648 & 0.533 & 1.352 & 1.227 & 0.907 & 2.024 & 20.16 & 0.533 & 193 & 9.58 & 0.798 \\
\hline UGC 11748 & 54.2 & 0.581 & 0.576 & 0.992 & 2.024 & 3.07 & 54.2 & 177 & 57.5 & 0.610 \\
\hline UGC 11819 & 0.307 & 1.235 & 1.084 & 0.878 & 2.024 & 43.0 & 0.307 & 328 & 7.62 & 3.90 \\
\hline
\end{tabular}

and from the best fits, the values of $r_{\epsilon}$ do not correspond to $r_{\epsilon}>3 r_{c}$ (Schive et al. 014a). The transition from the soliton to the NFW profile is smoother in this case, and that behavior is maintained as in the continuity condition analysis (see Section 4.1). From the combined analysis of the 18 LSB galaxies, we obtained an ultra-light mass $m_{\psi}=$ $2.024 \times 10^{-23} \mathrm{eV}$, that is in agreement with the cosmological constraints (Bozek et al. 2015; Sarkar et al. 2016), unlike the results with the continuity condition only.

Finally, in Table B3 we show the results from the individual analysis of the $6 \mathrm{NGC}$ galaxies with photometric information. In this case, the boson mass is in the range $0.264 \leqslant m_{\psi} / \mathrm{eV} \leqslant 30.0$, core radius within $0.311 \leqslant r_{c} / \mathrm{kpc} \leqslant$ 4.84 and ratio between the transition and core radii in 


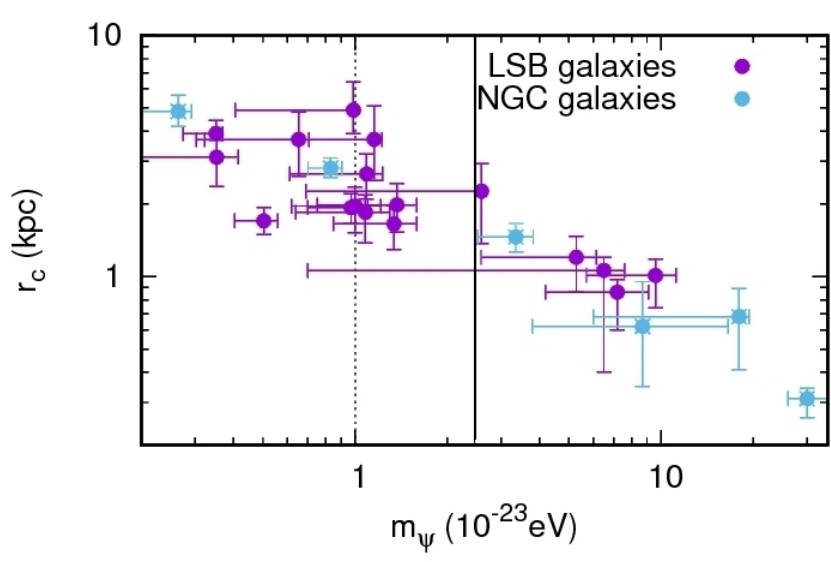

Figure B1. Distribution of resulting core radii $r_{c}$ vs. boson masses $m_{\psi}$ from the soliton+NFW density profile in the FDM model with the differentiability condition. The data is from the individual analysis of the 18 LSB and 6 NGC (including baryonic information) galaxies, $\pm 1 \sigma$ errors from the MCMC method. The vertical dashed line at $m_{\psi}=10^{-23} \mathrm{eV}$ shows the cosmological constraint (Bozek et al. 2015; Sarkar et al. 2016). The vertical solid line at $m_{\psi}=2.024 \times 10^{-23} \mathrm{eV}$ shows the resulting boson mass from the combined analysis of the 18 LSB galaxies.

$0.882 \leqslant r_{\epsilon} \leqslant 1.157$. Fig. B1 shows the distribution of $r_{c}$ vs. $m_{\psi}$ for all the LSB and NGC galaxies.

\section{References}

Alcubierre M., Guzman F. S., Matos T., Nunez D., Ureña-López L. A., Wiederhold P., 2002, Class. Quant. Grav., 19, 5017

Arbey A., Lesgourgues J., Salati P., 2001, Phys. Rev. D, 64, 123528

Arbey A., Lesgourgues J., Salati P., 2002, Phys. Rev., D65, 083514

Armengaud E., Palanque-Delabrouille N., Yèche C., Marsh D. J. E., Baur J., 2017, preprint (arXiv:1703.09126)

Avilez A. A., Padilla L. E., Bernal T., Matos T., 2017, preprint (arXiv: 1704.07314)

Baldeschi M. R., Ruffini R., Gelmini G. B., 1983, Phys. Lett., B122, 221

Bennett C. L., et al., 2013, The Astrophysical Journal Supplement Series, 208, 20

Bernal A., Matos T., Nunez D., 2008, Rev. Mex. A.A., 44 No. 1, 149

Bernal A., Barranco J., Alic D., Palenzuela C., 2010, Phys. Rev. D, 81, 044031

Bernal T., Robles V. H., Matos T., 2017, MNRAS, 468, 3135

Bertone G., Hooper D., Silk J., 2005, Phys. Rep., 405, 279

Boehmer C. G., Harko T., 2007, JCAP, 0706, 025

Boylan-Kolchin M., Bullock J. S., Kaplinghat M., 2011, MNRAS, 415, L40

Bozek B., Marsh D. J. E., Silk J., Wyse R. F. G., 2015, Mon. Not. Roy. Astron. Soc., 450, 209

Bray H. L., 2010, preprint (arXiv:1004.4016)

Bray H. L., 2012, preprint (arXiv:1212.5745)

Brun R., Rademakers F., 1997, Nuclear Instruments and Methods in Physics Research A, 389, 81

Burkert A., 1996, IAU Symp., 171, 175

Calabrese E., Spergel D. N., 2016, MNRAS, 460, 4397

Caputi K. I., et al., 2015, Astrophys. J., 810, 73

Chavanis P.-H., 2016, Phys. Rev. D, 94, 083007

Chen S.-R., Schive H.-Y., Chiueh T., 2017, MNRAS, 468, 1338
Davé R., Spergel D. N., Steinhardt P. J., Wandelt B. D., 2001, ApJ, 547, 574

Del Popolo A., 2009, ApJ, 698, 2093

Del Popolo A., 2012a, MNRAS, 419, 971

Del Popolo A., 2012b, MNRAS, 424, 38

Del Popolo A., Le Delliou M., 2017, Galaxies, 5, 17

Di Cintio A., Brook C. B., Macciò A. V., Stinson G. S., Knebe A., Dutton A. A., Wadsley J., 2014, MNRAS, 437, 415

Diez-Tejedor A., Gonzalez-Morales A. X., Profumo S., 2014, Phys. Rev., D90, 043517

Dutta P., Begum A., Bharadwaj S., Chengalur J. N., 2009, MNRAS, 398, 887

El-Zant A., Shlosman I., Hoffman Y., 2001, ApJ, 560, 636

El-Zant A. A., Hoffman Y., Primack J., Combes F., Shlosman I., 2004, ApJ, 607, L75

Elbert O. D., Bullock J. S., Garrison-Kimmel S., Rocha M., Oñorbe J., Peter A. H. G., 2015, MNRAS, 453, 29

Fernandez-Hernandez L. M., Rodriguez-Meza M. A., Matos T., 2017, preprint, (arXiv:1708.06681)

Gamerman D., 1997, Markov Chain Monte Carlo: Stochastic Simulation for Bayesian Inference. Chapman \& Hall/CRC Texts in Statistical Science, Taylor \& Francis

Garcia-Aspeitia M. A., Lopez-Dominguez J. C., Ortiz C., Hinojosa-Ruiz S., Rodriguez-Meza M. A., 2017, Revista Mexicana de Fisica, 63, 423

Gelman A., Rubin D. B., 1992, Statistical Science, 7, 457

Gentile G., Famaey B., Combes F., Kroupa P., Zhao H. S., Tiret O., 2007, Astron. Astrophys., 472, L25

González-Morales A. X., Marsh D. J. E., Peñarrubia J., UreñaLópez L., 2016, preprint, (arXiv: 1609.05856)

Goodman J., 2000, New Astron., 5, 103

Governato F., et al., 2010, Nature, 463, 203

Guzmán F. S., Lora-Clavijo F. D., 2015, Gen. Rel. Grav., 47, 21

Guzman F. S., Matos T., Villegas H. B., 1999, Astron. Nachr., 320,97

Harko T., 2011, JCAP, 1105, 022

Hernández-Almada A., Rodríguez-Meza M. A., Matos T., 2011, in Ureña-López L. A., Aurelio Morales-Técotl H., LinaresRomero R., Santos-Rodríguez E., Estrada-Jiménez S., eds, American Institute of Physics Conference Series Vol. 1396, American Institute of Physics Conference Series. pp 196-201, doi:10.1063/1.3647547

Hlozek R., Grin D., Marsh D. J. E., Ferreira P. G., 2015, Phys. Rev., D91, 103512

Hu W., Barkana R., Gruzinov A., 2000, Physical Review Letters, 85,1158

Hui L., Ostriker J. P., Tremaine S., Witten E., 2017, Phys. Rev. D, 95, 043541

Ibata R. A., et al., 2013, Nature, 493, 62

Ibata N. G., Ibata R. A., Famaey B., Lewis G. F., 2014, Nature, 511,563

Iršič V., Viel M., Haehnelt M. G., Bolton J. S., Becker G. D., 2017, preprint (arXiv:1703.04683)

Ji S. U., Sin S. J., 1994, Phys. Rev. D, 50, 3655

Klypin A., Kravtsov A. V., Valenzuela O., Prada F., 1999, ApJ, 522,82

Koda J., et al., 2015, ApJ, 802, L24

Kolb E., Turner M., 1994, The Early Universe. Frontiers in physics, Westview Press

Kroupa P., 2012, Publ. Astron. Soc. Austral., 29, 395

Kroupa P., et al., 2010, A\&A, 523, A32

Lee J.-w., Koh I.-g., 1996, Phys. Rev., D53, 2236

Lelli F., McGaugh S. S., Schombert J. M., 2016, AJ, 152, 157

Lelli F., McGaugh S. S., Schombert J. M., Pawlowski M. S., 2017, ApJ, 836, 152

Li B., Rindler-Daller T., Shapiro P. R., 2014, Phys. Rev. D, 89, 083536 
Table B3. NGC galaxies with the soliton+NFW profile with differentiability condition. In this Table we show the fitting parameters $\rho_{c}, r_{c}$ and $r_{\epsilon}$, the resulting boson mass $m_{\psi}$, the concentration parameters $c$ and $r_{200}$ radii from the NFW profile, and the $\chi_{\text {red }}^{2}$ errors for the soliton+NFW profile, for the NGC galaxies with photometric information. In the top panel we show the DM-only fits and in the bottom panel the fits taking into account the baryonic contribution.

\begin{tabular}{|c|c|c|c|c|c|c|c|c|}
\hline \multicolumn{9}{|c|}{ DM-only fits } \\
\hline Galaxy & $\begin{array}{c}\rho_{c} \\
\left(10^{-2} M_{\odot} / \mathrm{pc}^{3}\right)\end{array}$ & $\begin{array}{c}r_{c} \\
(\mathrm{kpc})\end{array}$ & $\begin{array}{c}r_{\epsilon} \\
(\mathrm{kpc})\end{array}$ & $r_{\epsilon} / r_{c}$ & $\begin{array}{c}m_{\psi} \\
\left(10^{-23} \mathrm{eV}\right)\end{array}$ & $c$ & $\begin{array}{l}r_{200} \\
(\mathrm{kpc})\end{array}$ & $\chi_{\mathrm{red}}^{2}$ \\
\hline NGC7814 & $4730 \pm 4.8$ & $0.0872_{-0.0067}^{+0.010}$ & $0.0774_{-0.0063}^{+0.0096}$ & 0.888 & $27.0_{-5.5}^{+2.8}$ & 69.425 & 147.32 & 5.541 \\
\hline NGC6503 & $1430_{-130}^{+190}$ & $0.0464_{-0.0028}^{+0.0036}$ & $0.0402_{-0.0025}^{+0.0032}$ & 0.866 & $171 \pm 20$ & 28.59 & 98.725 & 6.38 \\
\hline NGC3741 & $133_{-20}^{+13}$ & $0.0367 \pm 0.0050$ & $0.0315 \pm 0.0043$ & 0.858 & $932_{-300}^{+200}$ & 6.032 & 72.329 & 0.646 \\
\hline NGC1003 & $90_{-24}^{+52}$ & $0.154_{-0.065}^{+0.046}$ & $0.133_{-0.056}^{+0.040}$ & 0.864 & $69_{-30}^{+70}$ & 8.778 & 124.246 & 4.864 \\
\hline NGC1560 & $5.63_{-0.88}^{+0.64}$ & $\begin{array}{l}1.39 \pm 0.065 \\
1.39\end{array}$ & $\begin{array}{l}-0.056 \\
1.31 \pm 0.20\end{array}$ & 0.942 & $3.16_{-0.78}^{+0.50}$ & 7.983 & 95.974 & 0.395 \\
\hline NGC6946 & $5.14_{-0.47}^{+0.32}$ & $4.92_{-0.30}^{+0.36}$ & $6.28 \pm 0.70$ & 1.276 & $\begin{array}{c}0.14_{-0.78}^{+0.019} \\
0.254_{-0.029}^{+0.02}\end{array}$ & 27.294 & 150.211 & 1.616 \\
\hline \multicolumn{9}{|c|}{ DM+baryons fits } \\
\hline NGC7814 & $5.57_{-1.4}^{+0.66}$ & $4.84_{-0.66}^{+0.80}$ & $5.6 \pm 1.2$ & 1.157 & $0.264_{-0.070}^{+0.028}$ & 18.492 & 177.278 & 0.86 \\
\hline NGC6503 & $\begin{array}{l}4.59_{-0.68}^{-0.4} \\
+0.41\end{array}$ & $\begin{array}{r}-0.66 \\
2.81_{-0.24}^{+0.29}\end{array}$ & $3.07_{-0.34}^{+0.38}$ & 1.093 & $0.831_{-0.13}^{+0.070}$ & 13.809 & 106.975 & 1.547 \\
\hline NGC3741 & $6.33_{-2.9}^{+0.68}$ & $0.68_{-0.27}^{+0.21}$ & $0.60_{-0.25}^{+0.34}$ & 0.882 & $18.0_{-12}^{+1.43}$ & 4.687 & 93.217 & 2.115 \\
\hline NGC1003 & $23.1_{-3.2}^{+6.1}$ & $0.311_{-0.052}^{+0.033}$ & $0.269_{-0.045}^{+0.029}$ & 0.865 & $30.0_{-4}^{+8}$ & 5.062 & 135.755 & 2.93 \\
\hline NGC1560 & $4.11_{-0.65}^{+0.42}$ & $1.46 \pm 0.20$ & $1.38_{-0.23}^{+0.040}$ & 0.945 & $3.35_{-0.83}^{+0.47}$ & 7.113 & 86.89 & 0.234 \\
\hline NGC6946 & $27_{-12}^{+16}$ & $0.62_{-0.27}^{+0.33}$ & $0.55_{-0.24}^{+0.30}$ & 0.887 & $\begin{array}{l}8.7_{-4.9}^{+7.9} \\
\end{array}$ & 9.376 & 143.84 & 5.34 \\
\hline
\end{tabular}

Li B., Shapiro P. R., Rindler-Daller T., 2016, preprint, (arXiv: 1611.07961)

Lora V., Magaña J., 2014, J. Cosmology Astropart. Phys., 9, 011

Lora V., Magaña J., Bernal A., Sánchez-Salcedo F. J., Grebel E. K., 2012, J. Cosmology Astropart. Phys., 2, 011

Lovell M. R., et al., 2012, MNRAS, 420, 2318

Ludlow A. D., et al., 2017, Physical Review Letters, 118, 161103

Lundgren A. P., Bondarescu M., Bondarescu R., Balakrishna J., 2010, ApJ, 715, L35

Ma C.-P., Boylan-Kolchin M., 2004, Physical Review Letters, 93, 021301

Madau P., Shen S., Governato F., 2014, ApJ, 789, L17

Magaña J., Matos T., Robles V., Suárez A., 2012, Journal of Physics Conference Series, 378, 012012

Marsh D. J. E., 2016, Phys. Rep., 643, 1

Marsh D. J. E., Ferreira P. G., 2010, Phys. Rev. D, 82, 103528

Marsh D. J. E., Pop A.-R., 2015, Mon. Not. Roy. Astron. Soc., 451,2479

Martinez-Medina L. A., Matos T., 2014, Mon. Not. Roy. Astron. Soc., 444,185

Martinez-Medina L. A., Robles V. H., Matos T., 2015a, Phys. Rev. D, 91, 023519

Martinez-Medina L. A., Bray H. L., Matos T., 2015b, JCAP, 1512,025

Matos T., Guzman F. S., 2000, Class. Quant. Grav., 17, L9

Matos T., Robles V. H., 2016, preprint (arXiv:1601.01350)

Matos T., Rodríguez-Meza M. A., 2014, Journal of Physics Conference Series, 545, 012009

Matos T., Suarez A., 2011, Europhys. Lett., 96, 56005

Matos T., Ureña-López L. A., 2000, Classical and Quantum Gravity, $17, \mathrm{~L} 75$

Matos T., Ureña-López L. A., 2001, Phys. Rev., D63, 063506

Matos T., Ureña-López L. A., 2007, Gen. Rel. Grav., 39, 1279

Matos T., Guzmán F. S., Ureña-López L. A., 2000, Class. Quant. Grav., 17, 1707

Matos T., Vázquez-González A., Magaña J., 2009, MNRAS, 393, 1359

Matos T., Avilez A., Bernal T., Chavanis P.-H., 2016, preprint, (arXiv: 1608.03945)

McGaugh S. S., 2005, The Astrophysical Journal, 632, 859
McGaugh S. S., de Blok W. J. G., Schombert J. M., Kuzio de Naray R., Kim J. H., 2007, ApJ, 659, 149

McGaugh S. S., Lelli F., Schombert J. M., 2016, Physical Review Letters, 117, 201101

Membrado M., Pacheco A. F., Sañudo J., 1989, Phys. Rev., A39, 4207

Mocz P., Vogelsberger M., Robles V., Zavala J., Boylan-Kolchin M., Hernquist L., 2017, preprint, (arXiv: 1705.05845)

Monachesi A., Bell E. F., Radburn-Smith D. J., Bailin J., de Jong R. S., Holwerda B., Streich D., Silverstein G., 2016, MNRAS, 457,1419

Moore B., Quinn T., Governato F., Stadel J., Lake G., 1999, MNRAS, 310, 1147

Navarro J. F., Frenk C. S., White S. D. M., 1997, Astrophys. J., 490,493

Navarro J. F., et al., 2010, MNRAS, 402, 21

Nipoti C., Binney J., 2015, MNRAS, 446, 1820

Nipoti C., Treu T., Ciotti L., Stiavelli M., 2004, MNRAS, 355, 1119

Núñez D., González-Morales A. X., Cervantes-Cota J. L., Matos T., 2010, Phys. Rev. D, 82, 024025

Pawlowski M. S., Pflamm-Altenburg J., Kroupa P., 2012, Mon. Not. Roy. Astron. Soc., 423, 1109

Pawlowski M. S., et al., 2014, MNRAS, 442, 2362

Peebles P. J. E., 2000, ApJ, 534, L127

Planck Collaboration et al., 2016, Astron. Astrophys., 594, A13

Pontzen A., Governato F., 2012, MNRAS, 421, 3464

Pontzen A., Governato F., 2014, Nature, 506, 171

Press W. H., Ryden B. S., Spergel D. N., 1990, Phys. Rev. Lett., 64,1084

Robles V. H., Matos T., 2012, Mon. Not. Roy. Astron. Soc., 422, 282

Robles V. H., Matos T., 2013a, Phys. Rev. D, 88, 083008

Robles V. H., Matos T., 2013b, ApJ, 763, 19

Robles V. H., Lora V., Matos T., Sánchez-Salcedo F. J., 2015, ApJ, 810, 99

Robles V. H., et al., 2017, preprint, (arXiv:1706.07514)

Rodríguez-Meza M. A., Hernández-Almada A., Matos T., 2012, Revista Mexicana de Fisica E, 58, 53

Rodríguez-Montoya I., Magaña J., Matos T., Pérez-Lorenzana A., 
2010, ApJ, 721, 1509

Romano-Díaz E., Shlosman I., Hoffman Y., Heller C., 2008, ApJ, 685, L105

Rubin V. C., 1983, Science, 220, 1339

Rubin V. C., Ford W. K. J., . Thonnard N., 1980, ApJ, 238, 471

Sahni V., Wang L., 2000, Phys. Rev., 62, 103517

Salucci P., Burkert A., 2000, ApJ, 537, L9

Sarkar A., Mondal R., Das S., Sethi S. K., Bharadwaj S., Marsh D. J., 2016, Journal of Cosmology and Astroparticle Physics, 2016, 012

Sawala T., et al., 2016, MNRAS, 457, 1931

Schive H.-Y., Chiueh T., Broadhurst T., Huang K.-W., 2016, ApJ, 818,89

Schive H.-Y., Chiueh T., Broadhurst T., 2014a, Nature Phys., 10, 496

Schive H.-Y., Liao M.-H., Woo T.-P., Wong S.-K., Chiueh T., Broadhurst T., Hwang W. Y. P., 2014b, Phys. Rev. Lett., 113,261302

Schwabe B., Niemeyer J. C., Engels J. F., 2016, Phys. Rev. D, 94, 043513

Sin S.-J., 1994, Phys. Rev., D50, 3650

Smith S., 1936, ApJ, 83, 23

Spergel D. N., Steinhardt P. J., 2000, Physical Review Letters, 84,3760

Strigari L. E., Bullock J. S., Kaplinghat M., Simon J. D., Geha M., Willman B., Walker M. G., 2008, Nature, 454, 1096

Suárez A., Chavanis P.-H., 2017, Phys. Rev. D, 95, 063515

Suarez A., Matos T., 2011, Mon. Not. Roy. Astron. Soc., 416, 87

Suárez A., Robles V. H., Matos T., 2014, Astrophysics and Space Science Proceedings, 38, 107

Teyssier R., Pontzen A., Dubois Y., Read J. I., 2013, MNRAS, 429, 3068

Ureña-López L. A., Bernal A., 2010, Phys. Rev. D, 82, 123535

Ureña-López L. A., González-Morales A. X., 2016, JCAP, 7, 048

Ureña-López L. A., Robles V. H., Matos T., 2017, preprint, (arXiv:1702.05103)

Walker M., 2013, Dark Matter in the Galactic Dwarf Spheroidal Satellites. Springer Science+Business Media Dordrecht, p. 1039, doi:10.1007/978-94-007-5612-0_20

Weinberg D. H., Bullock J. S., Governato F., Kuzio de Naray R., Peter A. H. G., 2015, Proceedings of the National Academy of Science, 112, 12249

Wetterich C., 2001, Physics Letters B, 522, 5

Woo T.-P., Chiueh T., 2009, ApJ, 697, 850

Yoshida N., Springel V., White S. D. M., Tormen G., 2000, ApJ, 544, L87

Zavala J., Jing Y. P., Faltenbacher A., Yepes G., Hoffman Y., Gottlöber S., Catinella B., 2009, ApJ, 700, 1779

Zhao H., 1996, Mon. Not. Roy. Astron. Soc., 278, 488

Zwicky F., 1933, Helvetica Physica Acta, 6, 110

Zwicky F., 1937, ApJ, 86, 217

de Blok W. J. G., McGaugh S. S., Rubin V. C., 2001, AJ, 122, 2396

This paper has been typeset from a $\mathrm{T}_{\mathrm{E}} \mathrm{X} / \mathrm{AAT}_{\mathrm{E}} \mathrm{X}$ file prepared by the author. 